\title{
4. Bestandsaufnahme vor Ort: Erfassung und erste Aufbauarbeiten
}

Am Morgen des 22. Juni 1941 fielen die deutschen Truppen in die Sowjetunion ein ${ }^{1}$. Bei ihrem Vormarsch im russischen Raum sollten sie neben Völkerschaften und Gruppen verschiedenster Nationalitäten auch bald auf deutschstämmige Sowjetbürger treffen. Die meist in Siedlungen zusammenlebenden Deutschrussen wurden unverzüglich von verschiedenen Dienststellen und Stäben in mehreren aufeinanderfolgenden Stufen kenntlich gemacht, erfaßt und in besonderer Weise betreut. Eine erste provisorische Bestandsaufnahme nahmen die Feldkommandanturen der deutschen Wehrmacht noch im Operationsbereich und im rückwärtigen Heeresgebiet vor. Es folgten die Maßnahmen „zur Stärkung des deutschen Volkstums" von seiten der Einsatzgruppen der Sicherheitspolizei und des SD. Unterstützt und dann abgelöst bei der „Betreuungsarbeit“ im rückwärtigen Heeresgebiet und in dem an die Zivilverwaltung übergehenden Gebieten wurden die Einsatzgruppen von den Sonderkommandos der Volksdeutschen Mittelstelle. Unter der Zivilverwaltung führten außerdem zivile Stäbe, so ein Sonderkommando des Reichsministeriums Ost, spezielle Erfassungsaufgaben durch.

\section{Die Maßnahmen der Wehrmacht}

Die Angehörigen der Wehrmacht wurden in den ersten Monaten ihres Einsatzes in der UdSSR durch besondere Richtlinien auf das Vorhandensein einer deutschen Minderheit im Lande aufmerksam gemacht; diese Anweisungen bestimmten zugleich das Verhalten der Wehrmacht gegenüber der gesamten im Ostraum angetroffenen Bevölkerung². Sie nannten als Ziel des deutschen Feldzugs gegen das ,jüdisch-bolschewistische System“ die „völlige Zerschlagung der Machtmittel und die Ausrottung des asiatischen Einflusses im europäischen Kulturkreis“. Unter dieser Zielsetzung sollte sich der deutsche Soldat im Ostraum der Leitidee bewußt sein, daß er „Träger einer unerbittlichen völkischen Idee und Rächer für alle Bestialitäten [sei], die deutschem und artverwandtem Volkstum zugefügt wurden". Seine geschichtliche Aufgabe sollte der deutsche Soldat darin sehen, das ganze deutsche Volk ,von der asiatischjüdischen Gefahr ein für allemal zu befreien“. Damit war auch seine Rolle gegenüber

1 Aus sowjetischer Sicht: Istorija Velikoj Otečestvennoj Vojny Sovetskogo Sojuza 1941-1945 g., Moskva 1965; Nekritsch, A., Grigoreno, P., Genickschuß; die Rote Armee am 22. Juni 1941, Wien 1969. Zum diplomatischen Hintergrund aus deutscher Sicht: Sommer, E. F., Das Memorandum. Wie der Sowjetunion der Krieg erklärt wurde, München 1981.

2 „Verhalten der Truppe im Ostraum, Nbg. Dok. USSR-10, Armee-Oberkommando Ost 6, Abt. Ia - Az.7, A.H.Qu., 10. Oktober 1941. 
den in der Sowjetunion lebenden Deutschen als die eines Schutzpatrons und zugleich eines Rächers vorgezeichnet.

Den ersten Deutschrussen begegneten die deutschen Frontverbände noch vor Erreichen der deutschen Siedlungen. Die Luftwaffe warf über den sowjetischen Kampfverbänden Flugblätter in Russisch und Deutsch mit der Aufforderung zum Überlaufen ab. Neben den Parolen „Štyky v zemlju!“ („Bajonette in die Erde!“, d.h. „Dem Krieg ein Ende!", ein Slogan der Bolschewiki aus dem Ersten Weltkrieg) und „Stalin kaput!" enthielten die Flugblätter einen „Passierschein“ zum Überschreiten der Grenzlinie. Den Überläufern wurde Anonymität und gute Behandlung zugesichert ${ }^{3}$. Die Propagandaaktion verfehlte ihre Wirkung nicht. Mit den ukrainischen und russischen Deserteuren stellten sich auch bald die ersten deutschen ein - Bauernsöhne aus dem Wolgagebiet, der Ukraine, dem Kaukasus. Russische Bataillone, aus denen die Soldaten deutscher Herkunft desertiert waren, führten in einigen Fällen Vergeltungsmaßnahmen durch. So wurden in einer bei Pinica (Winniza, Podolien?) operierenden sowjetischen Kompanie als Strafe für die Desertion des Soldaten Weisfeld sieben andere Deutsche (unter ihnen Lubinskij, Gapa, Becker, Henke, Gorala und Junscynski) erschossen ${ }^{4}$.

Neben Überläufern deutscher Herkunft kamen auch volksdeutsche Angehörige eingekreister sowjetischer Truppenverbände in deutsche Kriegsgefangenschaft. Beide Gruppen wurden in den Lagern von den Gefangenen anderer Nationalitäten isoliert und auf Veranlassung des OKW in einem Sonderlager für deutschstämmige Kriegsgefangene in Preußisch-Stargard zusammengezogen. Sie sollten danach in einem Lager in der Nähe Oslos konzentriert werden ${ }^{5}$. Für die Dienststellen der Wehrmacht galt die Anordnung, daß volksdeutsche Kriegsgefangene wohl als Dolmetscher und ortskundige Führer, nicht aber als aktive Kämpfer eingesetzt werden durften ${ }^{6}$. Darüber hinaus erhoffte man sich von den ortskundigen Deutschen aus der UdSSR besondere Hinweise und Hilfsdienste zur Überführung von Partisanen. Für die Anzeige von Partisanen wurde eine Belohnung von 500 Rubel in Aussicht gestellt? Die Kriegsgefangenenlager wurden von den Einheiten der Sicherheitspolizei und des SD bewacht. Diese wählten, entgegen den Anordnungen des OKW, häufig als verläßlich geltende deutschstämmige Kriegsgefangene aus, um sie als Hilfskräfte zu besonderen Aufgaben zu verwenden. Sie konnten als Hilfsfreiwillige (Hiwis) bei der Lagerbewachung eingesetzt werden ${ }^{8}$ und hatten u. a. die Aufgabe, die eintreffenden Kriegsge-

${ }^{3}$ GR T 77, R 1006, F 2469921; vgl. auch Streit, Chr., Keine Kameraden. Die Wehrmacht und die sowjetischen Kriegsgefangenen 1941-1945, Stuttgart 1978.

${ }^{4}$ GR T 78, R 482, F 6466024 ff., Deutsche Heeresleitung, AOK, Aussagen eines Kriegsgefangenen, IIb, Nr. 4226, AOK 9 Ic/AO v. 11.10. 1944.

${ }^{5}$ Vgl. Referat D VIII 2723, Eing. 13. Oktober 1941, Direktorenkonferenz am 9.August 1941, AA Inl. II C, Akten betreffend: Förderung des Deutschtums in Rußland, Bd. 4 (1937-44), Nr.1.

${ }^{6}$ Nbg. Dok. NOKW-2115, 6. Panzer-Division/Ic, UdSSR, 23. Juli 1941, Ic-Nachrichtenblatt, Anweisung, Gen.Kdo. XXXXI A.K.

7 Ebenda.

${ }^{8}$ Nbg. Dok. NO-3372. 
fangenen nach Nationalität, politischen Ansichten, weltanschaulicher Glaubwürdigkeit usw. zu befragen und zu unterscheiden'. Hatten sie sich hier in den Augen der SD-Kommandos bewährt, konnten sie zu weiteren Einsätzen verwendet werden. So wurden in der Zeit vom 22. bis 25.10.1941 24 Deutsche aus der Ukraine über Wien in das Konzentrationslager Auschwitz geschickt, wo sie als Helfer der SS an der Lageraufsicht beteiligt waren ${ }^{10}$. Im KZ Auschwitz erwarben sich die ukrainischen Deutschen den Ruf besonderer Brutalität.

Allgemein setzte sich im ersten Jahr des Rußlandfeldzugs wohl die Praxis durch, die deutschstämmigen Kriegsgefangenen zu isolieren, sie in besseren Unterkünften zu halten und bei nächstbester Gelegenheit an ihre Heimatorte zurückkehren zu lassen. Die SS unterstütze diese Prozedur; da sie die Betreuung der Volksdeutschen in den geschlossenen Wohngebieten übernehmen sollte, war ihr daran gelegen, die Männer zurückzuführen, um die Dorfgemeinden möglichst vollzählig und „biologisch vollwertig" zu erhalten. Besondere Aktivitäten bei der Suche nach Volksdeutschen entwickelte die Volksdeutsche Mittelstelle der SS. So "betreute“ sie bereits in den Kriegsgefangenenlagern die isoliert untergebrachten Deutschen und bereitete sie ideologisch auf ihren künftigen Einsatz vor. Auch die in den Wehrmachtlazaretten liegenden deutschstämmigen Verwundeten suchte sie auf, um sie nach ihrer Genesung sofort in die Heimatgemeinden zurückführen zu können.

In den weiteren Kriegsjahren allerdings, besonders nach der Wende von Stalingrad im Winter 1942/43, wurden übergelaufene oder kriegsgefangene Volksdeutsche nach Befragung und Feststellung der politischen Zuverlässigkeit umgehend mit deutscher Uniform und dem entsprechenden Dienstgrad versehen und in die deutschen Kampfverbände gesteckt.

Auch die deutsche Abwehr nahm sich in einigen Fällen der übergelaufenen oder kriegsgefangenen deutschstämmigen Soldaten und Offiziere aus der Sowjetarmee an. So verfaßte der sowjetische Kriegsgefangene deutscher Herkunft Hauptmann Michael von Apen im Auftrag der Abwehr im Kriegsgefangenenlager Luft 2 in Lodz einen Aufruf an das russische Volk ${ }^{12}$, worin er die sowjetischen Kameraden zum Aufstand gegen "Juden- und Bolschewistenherrschaft" und zur Befreiung von Millionen unschuldiger Opfer dieses Regimes aufforderte.

Die in den Frontabschnitten operierende Abwehr (OKW/A Ausl/Abw Abt. Abw. III) warf auch zuerst die Frage auf, wer von den vorgefundenen deutschsprachigen Personen tatsächlich als Volksdeutscher bestimmt und anerkannt werden dürfe. Sie erbaten von Berlin eine Definition des Begriffs. In einer entsprechenden Verordnung des

9 Reitlinger, G., The House built on Sand. The Conflicts of German Policy in Russia, 1939-1945, London 1960, S.89; dt.: Ein Haus auf Sand gebaut. Hitlers Gewaltpolitik in Rußland, Hamburg 1962.

${ }^{10}$ Nbg. Dok. NO-3372-3374 und Hilberg, R., Destruction of the European Jews, Chicago 1961, S. 576.

${ }^{11}$ Referat D VIII 2799, Berlin, den 16. Oktober 1941, gez. Goeken, AA Inl. II C, Akten betreffend: Förderung des Deutschtums in Rußland, Bd. 4 (1937-44), Nr.1, D 626216.

12 Abwehrstelle im Wehrkreis XXI. Posen, den 29. Oktober 1942, GR T 77, R 1006, F 2470135 ff. 
Reichsinnenministers ${ }^{13}$ wurden alle jene Personen deutscher Abstammung als Volksdeutsche anerkannt, die den Nachweis führen konnten, daß mindestens drei Großelternteile „reine Deutsche“ waren. War jemand, obwohl er sich selbst zum Deutschtum bekannte, zum Erbringen des Nachweises nicht imstande, so konnte seine Anerkennung als Volksdeutscher "nach rassischer Überprüfung" erfolgen. Diese Anerkennung setzte freilich die Einschaltung der Rassenprüfer des Reichssicherheitshauptamtes bzw. der SS voraus. Als deutscher Volkszugehöriger durfte ferner anerkannt werden, „wer ohne der Abstammung nach zum deutschen Volkstum zu gehören, sich als Angehöriger der deutschen Nation bekennt, sofern dieses Bekenntnis durch Sprache, Haltung, Erziehung oder sonstige Umstände bestätigt ist oder glaubhaft gemacht wird und rassische Bedenken" gegen die entsprechende Person oder ihre Sippe nicht bestanden.

Der Generalstab des Heeres präzisierte bei der Bekanntgabe dieser Verordnung an alle Befehlshaber, daß „Fremdblütige“ selbst dann, wenn sie ein Bekenntnis zum Deutschtum ablegten, keinesfalls als Volksdeutsche anerkannt werden durften ${ }^{14}$; die deutsche Wehrmacht erkenne darüber hinaus nur solche Personen als Volksdeutsche an, „die sich bereits vor der Besetzung dieser Gebiete zum Deutschtum bekannt haben“. In demselben Schreiben wurde die Durchführung des Volkslistenverfahrens in Aussicht gestellt. Der Generalstab wies ferner erneut darauf hin, daß den Volksdeutschen eine bevorzugte Behandlung zustehe, warnte aber zugleich vor feindlichen Ausländern und Agenten, die sich als Volksdeutsche tarnten, um sich Vorteile zu verschaffen und bei Wehrmachtsdienststellen eingestellt zu werden. Eine Indienstnahme vermeintlicher Volksdeutscher erfordere „in jedem Fall die Entscheidung des zuständigen Regiments- oder selbständigen Bataillons- bzw. Abteilungskommandeurs oder des zuständigen Dienststellenleiters“.

Wo die Wehrmacht mehr oder minder geschlossene deutsche Siedlungen vorfand, bestimmten die Feldkommandanturen Bürgermeister und Vertrauensleute aus den Reihen der Volksdeutschen, die mit Registrierungsarbeiten und der Wirtschaftsführung der Gemeinden beauftragt wurden.

In den rückwärtigen Heeresgebieten setzten erste Maßnahmen zur Besserstellung und Förderung der deutschstämmigen Bevölkerungsteile ein. So sah die Anordnung des Befehlshabers des rückwärtigen Heeresgebiets Süd vom 16.8. $1941^{15}$ folgende Schritte zur „Unterstützung notleidender Volksdeutscher" vor: „Volksdeutsche sind bei Besetzung von Stellen in Wirtschaft und Verwaltung besonders zu berücksichtigen. Sie wenden sich an von hier eingesetzte Vertrauensmänner; Name und Einsatzort werden von Fall zu Fall bekanntgegeben. Volksdeutschen Siedlern ist durch Kredite der Gemeinden (sic) zu helfen; bis zum Erlaß weiterer Bestimmungen können

${ }^{13}$ GR T 454, R 20, F 386.

14 GenStdH/Gen Qu., Az. Abt. K. Verw (V), Nr.II/4775/41 geh., 5. 8. 1941, Betr.: Behandlung von Volksdeutschen, GR T 454, R 20, F $383 \mathrm{f}$.

15 Abt. VII, Nr.7, Befehlshaber des Rückwärtigen Heeresgebietes Süd, Nr.103/41, 16. 8. 41, Nbg. Dok. NOKW-1691. 
Vorschüsse gewährt werden. " Diese Anordnung hatte unter anderem zur Folge, daß in ethnisch gemischten Gemeinden die Gemeindekassen gänzlich an die deutschen Bevölkerungsteile ausgeschüttet wurden. Somit etablierte sich bereits unter der deutschen Wehrmachtführung das Prinzip der krassen nationalen Ungleichheit, das die nachrückenden SS-Kommandos bis zur massenvernichtenden Perfektion ausbauten. Die ersten größeren Komplexe zusammenhängender deutscher Siedlungen wurden von der Abwehr II bei der Heeresgruppe Süd im ehemals russisch-wolhynischen Gebiet (dem Gouvernement Wolhynien) ausgemacht ${ }^{16}$. Sie lagen im Städtedreieck Schitomir-Iskorost (deutsch: Korosten)-Nowograd-Wolynsk (Zwiahel). Hier hatten nach Ende des Ersten Weltkrieges ca. 70-80000 deutsche Siedler gelebt. Ihre Zahl war nun um etwa ein Drittel reduziert.

Neben der Aussiedlung der Deutschen aus einem Grenzstreifen von etwa $100 \mathrm{~km}$ (der sog. Stalinlinie) in Richtung Murmansk zu Beginn des Jahres $1935^{17}$ hatten vor allem die Wellen der Deportation (Schwerpunkte 1933 und 1939), der Verschickung von „Kulaken“-Familien (Großbauern) nach Sibirien und Archangelsk (ab 1929) sowie der „freiwilligen Verbannung" in die großen Industriegebiete des Donbas und der Dnjepr-Region und die agrarischen Erschließungsgebiete Mittelasiens deutliche Spuren im demographischen Aufbau der deutschen Bevölkerung hinterlassen ${ }^{18}$. Innerhalb der Siedlungsgebiete hatte gleichzeitig eine starke Abwanderung vom Land in die Städte stattgefunden, die größere Anonymität und eine weniger harte Arbeitslast als das Leben auf den Kolchosen versprachen. Im Zuge der Landflucht waren aus vielen ehemals geschlossenen deutschen Siedlungen und Dörfern ethnisch gemischte Kolchosen geworden (in NS-Terminologie: Mischsiedlungen); das früher kompakte deutsche Siedlungswesen stellte sich nun zum Teil als „Streudeutschtum“ dar.

Aufgrund des Männermangels infolge von Deportation und Erschießungen war die Zahl der ethnisch gemischten Ehen (Mischehen) relativ hoch; die deutschen Frauen waren zum Teil mit Russen und Ukrainern, vorwiegend der Intelligenzschicht und höheren militärischen Rängen, verheiratet. Die Ehe mit einem Russen versprach in vielen Fällen Sicherheit vor Verfolgung („Wer mit einem Russen verheiratet war, hatte Ruhe"). Da deutsche Schulen bis 1938 bestanden hatten, waren deutsche Sprachkenntnisse noch weithin vorhanden. Ein deutsches Kulturbewußtsein oder gar eine „nationale deutsche Gesinnung" wurden allerdings selten und dann nur in verdeckter Form angetroffen. Das kirchliche Leben dieser vorwiegend protestantischen Kolonisten war zerstört, die Kirchen waren profanisiert, die Geistlichen liquidiert („getötet

${ }^{16}$ Abwehr II, Heeresgruppe Süd, Lage des Deutschtums im Gebiet von Shitomir (Ehemalig Russ.Wolhynien), O.U., den 15. August 1941, GR T 454, R 20, F 127-34; BA R 57/neu 924. Die Abwehr II hatte bereits 1940 militärische Einheiten aus russischsprechenden Soldaten und Offizieren (Ukrainer, Letten u. a.) aufgestellt. Sie rückten zusammen mit den Fronteinheiten in die Ukraine ein. Vgl. Reile, O., Geheime Ostfront. Die deutsche Abwehr im Osten 1921-1945, München 1963, S. $366 \mathrm{ff}$.

$17 \mathrm{Vgl}$. zu diesen Fragen meinen Aufsatz: „Unternehmen Barbarossa“ und die Zwangsaussiedlung der Deutschen in der UdSSR, in: VfZ 30 (1982), S. 299-321.

${ }_{18}$ Vgl. dazu die Tabellen in: Ereignismeldung UdSSR Nr.75, 6.9. 1941, S. 2 ff. 
oder verschickt"). Wehrmachtgeistliche besorgten auf Wunsch der örtlichen Bevölkerung die ersten kirchlichen Zeremonien seit vielen Jahren. Das religiöse Leben erwachte schnell.

Die wirtschaftliche Lage dieser Deutschen, in ihrer großen Mehrheit KolchoseMitglieder, war vergleichsweise erträglich. Die deutschen Häuser zeichneten sich durch Sauberkeit aus.

Die Haltung dieser leidgeprüften Menschen überraschte die Angehörigen der Abwehr: „Der auf der Bevölkerung lastende Terror war so gewaltig, daß er große Lükken in das soziologische Gefüge der Volksgruppe riß und tiefe Spuren in das Antlitz und in die Seele der Menschen eingrub, ohne jedoch ihre seelische Kraft zu brechen. Man trifft kaum auf Menschen, die stumpf und gleichgültig geworden sind! Sondern es spricht aus ihrem Wesen oft eine innere Erhabenheit über das irdische Schicksal." Die ersten Maßnahmen zur Stärkung der deutschen Gemeinden bestanden in der Übergabe der Kolchose- und Gemeindeführung sowie der Leitung der Erntearbeiten an „tatkräftige deutsche Männer“. Am Ort aufgestellte und zum Teil bewaffnete Milizen übernahmen den „Ernteschutz" und nahmen die Fahndung nach „versprengten Bolschewisten und Partisanen“ auf; sie hatten „dabei auch Erfolge zu verzeichnen“. Kommunistische Funktionäre waren unter den Deutschen Wolhyniens kaum anzutreffen: „Nur in ganz wenigen Fällen waren Deutsche Handlanger des bolschewistischen Systems. Diese Elemente haben sich rechtzeitig in Sicherheit gebracht." Bei der Registrierung der Deutschen durch die neueingerichteten Ortskommandanturen leisteten junge deutsche Männer „wertvolle Dolmetscher- und Führungsdienste“. Junge Burschen, die sich den durchziehenden Truppen zur Verfügung stellten, wurden als Kraftfahrer und Dolmetscher eingesetzt.

Im Rücken der deutschen Truppen besuchte der Verbindungsmann des Auswärtigen Amtes zum Oberkommando des Heeres, Dr. Walter von Hellenthal, die deutschen Siedlungen im Gebiet Wolhynien ${ }^{19}$. Er bestätigte, daß die „Volksdeutschen ... durchweg einen gesunden Eindruck [machten] und ... keine Degenerationserscheinungen auf[wiesen]". Doch konstatierte er unter den Deutschen des Gebiets von NowogradWolynsk schwere Not, während die Deutschen im Gebiet von Schitomir-Ikorost auf großen Kollektivwirtschaften unter ökonomisch erträglicheren Bedingungen lebten.

Zur Abwehr II gehörte auch die Admiral Canaris unterstehende Division Brandenburg $^{20}$. Dem Stab der „Brandenburger“ gehörten mehrere Offiziere aus deutschbaltischem Adel an, die über die notwendigen Landes- und Sprachkenntnisse verfügten ${ }^{21}$.

19 Der Vertreter des Auswärtigen Amtes beim Armeeoberkommando C, Nr. 417, A.H.Qu., den 6. August 1941, (anschließend an die Berichte) $397 \mathrm{~g}$ vom 17.7. und $404 \mathrm{~g}$ vom 30.7. 1941, Inhalt: Volksdeutsche in der Ukraine, AA Inl. II C, Akten betreffend: Förderung des Deutschtums in Rußland, Bd. 4 (1937-44), Nr.1.

${ }^{20}$ Vgl. Bertold, W., Division Brandenburg, die Haustruppe des Admirals Canaris, München 1977; Abshagen, K.H., Canaris, Patriot und Weltbürger, Stuttgart 1954.

${ }^{21}$ Vgl. Leverkuehn, P., Der geheime Nachrichtendienst der deutschen Wehrmacht im Kriege, Frankfurt/Main 1959, S. 127 ff., $135 \mathrm{ff}$. 
Wie andere Einheiten der Abwehr II führten auch die Brandenburger Frontaufklärung durch ${ }^{22}$. Beim Eindringen in das Schwarzmeergebiet wurden Teile der Division zum Einsatz im deutschen Siedlungsgebiet zwischen Tiraspol und Odessa, den sogenannten Kutschurganer Kolonien (Selz, Kandel, Baden, Straßburg u.a.) abgestellt. Unter ihnen befand sich das 6. Lehrregiment „Brandenburg z.b.V. 800“. Die Anordnung des Oberbefehlshabers der 11. Armee vom 9. August 1941 lautete auf Schutz der deutschen Bevölkerung gegen die Plünderungen der in diesem Raum operierenden rumänischen Armeeteile. Die „Brandenburger" weiteten ihre Unterstützung der einheimischen Deutschen auch auf andere Sektoren aus. So kümmerte sich der Arzt der Truppe, Dr. Ehreninger, um die medizinische Betreuung der Deutschen; er übernahm sowohl die chirurgische Versorgung der durch die Gefechte verletzten Mitglieder der deutschen Gemeinden als auch die Behandlung chronischer Krankheiten (vor allem Trachoma, Malaria, Tuberkulose und Unterernährung der Kinder) ${ }^{23}$.

Die in der Zentralukraine bis hin zum westlichen Dnjeprbogen operierende Panzergruppe 1 hatte die deutschen Orte im Raum von Nikopol-Saporoschje-Kriwoj Rog, ehemals besonders wohlhabende Siedlungen im Zentrum des Gouvernements Jekaterinoslaw, besetzt. Allein im westlichen Dnjepr-Knie befanden sich ca. 50 deutsche Orte mit etwa 50000 Einwohnern ${ }^{24}$. Diese Siedlungen schienen den Berichterstattern der Panzergruppe nach den Erfahrungen in der Westukraine mit ihrer mehr oder minder verstreut lebenden deutschen Bevölkerung von einzigartiger Geschlossenheit, sie hatten "Sprache und Volksbewußtsein erstaunlich gut bewahrt" und zeigten „trotz starker Inzucht keine Degenerationserscheinungen“. Für den Neuaufbau der Ukraine unter der Schirmherrschaft des Deutschen Reiches versprachen sie, einen brauchbaren Grundstock abzugeben. Im Gegensatz zu den westukrainischen Deutschen waren diese Deutschen der Wehrmacht freundlich, teilweise begeistert entgegengekommen und hatten ehrliche Freude über die deutsche Besetzung gezeigt. In Dankesbeweisen und Kundgebungen der Sympathie für die deutschen Truppen verdeutlichten sie, daß sie die Wehrmacht „als sich verwandt und als Befreier vom bolschewistischen Joch" betrachteten. Unaufgefordert distanzierten sie sich von der kommunistischen Partei und vom Sowjetstaat. „Ganz vereinzelt hatte es natürlich auch deutsche Agenten in der Partei gegeben. Diese waren aber schon kalt gestellt, ehe deutsche Truppen kamen." In einem Punkt hebt sich jedoch der Bericht der Panzergruppe 1 von sonstigen Meldungen ab: „Auffallend ist überall der $\mathrm{Haß}$ und die instinktive $\mathrm{Ab}$ lehnung gegen den Juden, die sich offensichtlich daraus erklärt, daß man in ihm den Exponenten des Bolschewismus sah, der auch meist Parteiführer im Dorf und Vorsit-

22 Vgl. Reile, Ostfront, S. $371 \mathrm{ff}$.

${ }^{23}$ Vgl. 6. Lehrregiment „Brandenburg“ z.b.V. 800, Unterarzt und Truppenarzt, O.U., den 26.8. 1941, Betr.: Erfahrung und Tätigkeitsbericht während des Einsatzes im volksdeutschen Gebiet nordwestlich Odessa, GR T 454, R 20, F $145 \mathrm{ff}$;; darauf handschriftl. Vermerk, vermutlich des Empfängers: „Das SS-Kommando Hoffmeyer steht jetzt im benachbarten Landau u. betreut die Gegend."

${ }^{24}$ Vgl. Panzergruppe 1, Abteilung Qu., Gr.H.Qu., den 24.9. 41, Das Deutschtum im Dnjepr-Gebiet, GR T 454, R 20, F 153-57. 
zender der Wirtschaftsorganisation war." Zwar waren im Rahmen der machiavellistischen, auf Schärfung der inneren, nationalen Gegensätze bedachten Politik Stalins zahlreiche Juden als Funktionäre in deutschen Siedlungsgebieten eingesetzt, doch hatte diese Praxis, soweit Rückschlüsse aus anderen früheren Berichten gezogen werden können, in der Masse der deutschen Bevölkerung nicht zu einer derart haßerfüllten Einstellung gegenüber den Juden geführt. Im Gegenteil, der grundlegende Mangel an Juden- und Slawenhaß in der deutschrussischen Bevölkerung war eine Erscheinung, gegen welche die Einsatzgruppen der Sicherheitspolizei und des SD mit nur mäßigem Erfolg ankämpften.

\section{Die Maßnahmen des Reichsministeriums für die besetzten Ostgebiete}

Die Männer des Reichsostministeriums waren zwar in hohem Maße an der Erfassung und Betreuung des Rußlanddeutschtums interessiert; doch waren ihre Dienststellen als zivile Instanzen in den ersten Monaten des Rußlandfeldzuges nicht befugt, in den besetzten Gebieten vor Ort tätig zu werden. Sie mußten die Übergabe der entsprechenden Gebiete an die Zivilverwaltung abwarten, bevor sie in aller Öffentlichkeit ihren Einsatz leisten konnten. Allerdings machten sie von der Möglichkeit Gebrauch, Männer ihres Vertrauens, die sich als Wehrmachtsangehörige im Operationsbereich und im rückwärtigen Heeresgebiet befanden, als Informanten und tatkräftige Helfer zu benutzen.

Zu diesen gehörte Feldwebel Dr. Hermann Maurer, im Zivilberuf Hauptabteilungsleiter im Deutschen Auslandsinstitut Stuttgart, dem u.a. die von Stumpp geleitete Forschungsstelle Rußlanddeutschtum unterstand. Maurer, später offiziell Verbindungsmann zwischen dem DAI und dem Reichsministerium Ost, lag mit seiner Kompanie in der Zeit vom 13. bis 25. August 1941 in den Kutschurganer Siedlungen des Schwarzmeergebietes. Nach Eintreffen im deutschen Dorf Baden hatte er seine Leute umgehend durch Vorträge über die Geschichte und gegenwärtige Lage der Kutschurganer Deutschen aufgeklärt.

Er nutzte dann die kurze Zeit der Stationierung, um in den umliegenden deutschen Dörfern die ersten „Aufbauarbeiten“ durchzuführen" ${ }^{25}$. Im Einvernehmen mit der Abteilung Leibbrandts im RMO ging es Maurer in erster Linie darum, die traditionellen Sonderformen der deutschen Siedlungen in Rußland, Selbstverwaltung und Kulturautonomie, wiederherzustellen.

Die Bevölkerung der Kutschurganer Siedlungen, Anfang des 19.Jahrhunderts aus dem Südwesten Deutschlands eingewandert, erschien Maurer noch immer „rein deutschblütig“; Mischehen waren selten. Juden, sowjetische Funktionäre und „mit ihnen belastete Deutsche" hatten die Siedlungen in ihrer Mehrheit vor dem deutschen Einmarsch verlassen. Die männliche Bevölkerung war durch Verbannung und Ver-

${ }^{25}$ Erfahrungs- und Tätigkeitsbericht des Feldw. Dr. Hermann Maurer (O.A.) über Aufbauarbeiten in den deutschen Dörfern des Kutschurganer Bezirks bei Odessa vom 13.8. 1941-28.8. 1941, GR T 454, R 20, F 359-70, und GR T 81, R 412, F 5157333-44. 
folgung stark dezimiert. Es herrschte starker „Frauenüberschuß“. Die Kinderzahl war „eingeschränkt, aber doch ansehnlich“. Der Gesundheitszustand der deutschen Bevölkerung war aufgrund fehlender ärztlicher Versorgung und mangelhafter Ernährung schlecht („allgemeine Magerkeit“). Viele Kinder litten an verschleppten Krankheiten.

Über die Einstellung zur Arbeit registrierte Maurer: „Fast restlose Zerrüttung des Arbeitsethos, Faulenzen und Zwingen zur Arbeit bei vielen ein Dauerzustand, Unpünktlichkeit, Auflehnung gegen Anordnungen zur Leistungssteigerung, Neigung zur Aneignung fremden Gutes, Mangel an Zusammenarbeit und genossenschaftlichem Denken. Das Arbeitsleben ist nahezu vollkommen bolschewisiert. Verschiedene Stufen der Zerstörung des Arbeitswillens durch Kollektivwirtschaft und Vorenthalten des Arbeitsertrages; besonders auffällige Verfallserscheinungen bei den jüngeren Jahrgängen. Viele Frauen verbringen ein müßiges Leben im Hause und verlassen sich auf die Arbeit der Männer."

Viele der von Maurer beschriebenen Verhaltensformen stellten in psychologischer Hinsicht eine Art passiven Widerstandes, bei oberflächlicher Anpassung an die herrschende Macht, dar, die sich die russischen Deutschen vermutlich seit den Jahren des großen Wirtschaftsterrors angeeignet hatten. Die Tatsache, daß sie sie auch unter der deutschen Besatzung beibehielten, zeigt zumindest eine Form von Unwillen und mangelnder Bereitschaft zur Zusammenarbeit.

Bei der Beurteilung der verschiedenen Altersklassen sah Maurer in den älteren Jahrgängen noch die „Erhaltung gewisser Sitten und Reste von bäuerlicher Lebensart". Die mittleren Jahrgänge erschienen ihm „durch die bolschewistischen Polizeimaßnahmen weitgehend gebrochen“, die jüngeren Jahrgänge „verwahrlost ... Die Jugend bis zum 25. Lebensjahr muß vollkommen umgestaltet werden." Dennoch fand Maurer ,in jedem Jahrgang ... erstaunliche Ausnahmen an Gesundheit, Charakter und Erziehung“. So habe die Leistung vieler Männer in der „Aufrechterhaltung des Bekenntnisses zum Deutschtum trotz Verfolgung und Terror, Verbannung und Tod“, die zahlreicher Frauen in der Bewahrung der deutschen Dörfer vor dem völligen Untergang bestanden. Auf diesen Ausnahmen sollte die Vermittlung der nationalsozialistischen Lebensform an diese Deutschen aufbauen.

Ein kulturelles Leben bestand nicht mehr. Kirchen waren seit 1935 in Klubs, Tanzsäle, Viehställe verwandelt, die Kirchtürme abgetragen, die Glocken zerschlagen. Die verwahrlosten Friedhöfe wurden als Viehweiden benutzt. Der Besitz der Familien beschränkte sich auf Wohnraum, Küche, Stall und ein kleines Stück Garten vor dem Haus. Ein Teil der Familien besaß daneben eine Kuh (die sog. Stalinkuh), ein Schwein, eine Ziege und einige Hühner. In den Häusern war der Hausrat der vorrevolutionären Zeit vorhanden, Neuanschaffungen hatten nicht stattgefunden. Die Mehrheit der Häuser war „heruntergekommen“. Stattlichere Häuser, die den früheren Wohlstand ihrer Besitzer hätten verraten können, waren zur Tarnung mit schmutzigem Lehm beschmiert. Die Kleidung der Bevölkerung war außerordentlich dürftig. „Der Großteil der Bevölkerung geht in Lumpen. Sorgsame haben ein gutes Kleidungsstück im Hintergrund behalten. Viele besitzen nur Hose und Hemd, die 
Mehrheit kein Schuhzeug, die Frauen nur ein Kleid. Die Kinder gehen überhaupt in Lumpen." Besonders verwahrlost wirke die Jugend durch die unordentlich getragenen sowjetischen Schildmützen. Alle Frauen trügen abgeschnittenes Haar, bedeckt durch das „Komsomol-Tuch“; nur ältere Frauen hätten das Kopftuch zum Teil noch „nach Art der deutschen Bäuerinnen“ umgebunden.

Die Aufbauarbeiten Maurers dienten einem zweifachen Ziel: Sie sollten einerseits die Grundlagen für das Eindringen des Nationalsozialismus legen und andererseits die verschütteten Werte der Vergangenheit neubeleben. Der erste Schritt zur Herstellung der ehemaligen Dorfstruktur war die Errichtung der Dorfverwaltung. Auf Bürgerversammlungen wurden durch Zuruf ein Schulze (Bürgermeister) und der Gemeinderat bestimmt, der sich aus einem Feldleiter, je einem Rat für Viehzucht, Schulwesen und Kirchenwesen, einem Kassenverwalter, einem Dorfschreiber und einem „Führer der deutschen Mannschaft", d.h. des volksdeutschen Selbstschutzes, zusammensetzte. Unbesetzt blieben vorerst die Ämter der Frauenführung und Jugenderziehung. Sie sollten auf nationalsozialistischen Grundsätzen basieren, deshalb kamen hier nur reichsdeutsche Kräfte in Frage. Ferner wurde in jedem Dorf ein dreiköpfiges Laiengericht einberufen, das Rechtsstreitigkeiten schlichten und entsprechende Strafen verhängen sollte. Am 16. August rief Maurer die gewählten Schulzen der Kutschurganer Siedlungen - mit Ausnahme des noch umkämpften Mannheim - in Baden zusammen. Hier wurde der Badener Schulze Hilzendeger zum Gebietsschulzen (in der früheren Terminologie der deutschen Siedlungskolonien: Oberschulze) gewählt. Dem Gebietsschulzen wurde ein Vertreter und ein Gebietsschreiber, ein gewisser Merdian, vermutlich z.T. armenischer Herkunft, beigegeben. Mit der offiziellen Bestätigung dieser Amtspersonen durch die „Hoheitsträger“, den Kompaniechef und den rumänischen Distriktchef Oberst Jonescu, war es Maurer gelungen, in diesem ländlichen Bereich erneut die Fundamente einer Selbstverwaltung zu legen, wie sie im 19. Jahrhundert bestanden hatte.

Eine zweite Reihe von Maßnahmen setzte auf wirtschaftlichem Gebiet ein. Sie betrafen vor allem die Sicherstellung der Ernte. Die rumänischen Behörden, denen dieser Teil der besetzten Sowjetunion zufallen sollte, hatten laut Dekret Abgaben von 50\% der Ernte gefordert. Bei den Besprechungen über die Verteilung der Ernte entstanden die ersten Schwierigkeiten. Die deutschen Bauern forderten mit großem Nachdruck die Aufhebung der Kollektivwirtschaften, Kolchosen wie Sowchosen. Die reichsdeutsche Wirtschaftsführung, vor allem Hermann Göring als Bevollmächtigter für den Vierjahresplan, hatte beschlossen, die Kollektivwirtschaft als nützlichste Arbeitsform zur Erzielung maximaler Erträge aufrechtzuerhalten. Es lag nun bei den Organen der deutschen Wehrmacht, den erwartungsvollen Bauern klarzumachen, daß eine Auflösung der Kollektive zunächst nicht durchführbar sei. In der Regel behalfen sie sich mit einem terminologischen Trugschluß: Sie erklärten den Bauern, daß die Ernteerträge der Kollektivwirtschaften jetzt „Gemeindevermögen“ seien und „nachher zur Verteilung an die Dorfgenossen zur Verfügung" stünden. Doch - mit den Worten Maurers - „nicht jeder bisherige Kollektivist sah das ein. Manche hätten gerne ihre eigene Ernte fortgeholt, wo es ihnen gerade beliebte." Um die Widerstände 
gegen die Aufrechterhaltung der Kollektivordnung zu brechen, wurden erste disziplinarische Maßnahmen ergriffen: „Es wurde die Arbeitspflicht für jeden ausgerufen, der nicht begründet verhindert war. Als Arbeitsgrundlage diente die bisherige Arbeitseinheit." Damit wurde aber auch die „Fron unter dem Kolchossystem" fortgesetzt, die nationalsozialistische Medien bis zu Beginn des Rußlandfeldzuges als ein Übel der Entmenschlichung dargestellt hatten. Wie auf der Kolchose galt weiter das Leistungsprinzip. Bei ungenügender Arbeit wurden Disziplinarstrafen verhängt: Sie reichten von Stockschlägen bis zum Einsperren bei schmaler Ration. Die Feldarbeiter wurden gleichzeitig „zur Pflichterfüllung und Leistungssteigerung“ ermahnt. „Zur Überwachung der Arbeit wurden die Feldleiter eingesetzt ... Es wurde ein strenges Verbot eigenmächtigen Vorgehens erlassen. "Über eine Aufteilung des Landes in Privatbesitz durfte ebensowenig gesprochen werden wie über den zukünftigen Einsatz dieser Menschen. Ein großer Teil der örtlichen Bevölkerung glaubte nicht an den endgültigen deutschen Sieg und begehrte die Aussiedlung an sichere Plätze in Deutschland. Diese Fragen mußten unbeantwortet bleiben: „Festlegungen irgend welcher Art werden abgelehnt. Es wird auf die Ankunft einer Kommission der Volksdeutschen Mittelstelle hingewiesen."

Parallel zu diesen Maßnahmen wurde die "Sicherung gemeindeeigener Werte" vorgenommen. Arbeitsgeräte, Maschinen und Vieh der Kolchosen, die zum Teil von den zurückflutenden Truppenteilen der Roten Armee, zum Teil auf Anordnung des NKWD von den ortsansässigen Männern nach Osten abgezogen worden waren, sollten zurückgeschafft werden. Viele der Gerätschaften und Viehherden waren von den Sowjets nur bis zum Westufer des Bug gebracht worden. In welchem Umfang diese Herden und landwirtschaftlichen Maschinen (vor allem Dreschmaschinen, Mähdrescher und Traktoren) bei der Rückführungsaktion als zu den deutschen Dörfern gehörig identifiziert werden konnten oder ukrainische und russische Kollektivwirtschaften in Mitleidenschaft gezogen wurden, bleibt eine offene Frage.

Weitere Schritte zur Förderung des Siedlungsgebiets waren die Inbetriebnahme sowjetischer Musterbetriebe wie Weinkombinate oder Fabriken zur Herstellung von Wagen, Holz- und Korbwaren durch Deutsche, und auf kulturellem Gebiet Vorbereitungen zur Öffnung deutscher Schulen und Kirchen. Schließlich wurden die deutschen Dörfer durch besondere Beschilderung unter den Schutz der deutschen Wehrmacht gestellt, ein System verbindlicher Lebensmittelpreise festgelegt (das deutschen Siedlern auf den örtlichen Märkten große Vorteile verschaffte) und die Aufklärungsund Erziehungsarbeit unter Frauen und Jugendlichen begonnen.

Nach Abschluß seiner Tätigkeit stellte Maurer fest, daß die Einstellung zur Arbeit positiver und die Zahl der Unwilligen kleiner geworden sei. Die Führer der Dörfer gewannen, „sei es durch Härte, sei es durch gutes Zureden“, wieder an Autorität und Einfluß. Nach etwa einem Jahr berichteten der Gebietsschulze Hilzendeger und sein Schreiber Merdian in einem Schreiben an Maurer von dem „vollblütig strotzenden Leben " in den Kutschurganer Gemeinden ${ }^{26}$.

${ }^{26}$ GR T 454, R 20, F $371 \mathrm{ff}$. 
Während Maurer seine „Aufbauarbeiten“ durchführte, war seinem Bericht zufolge in denselben deutschen Siedlungen bereits ein "Sonderkommando der SS“ tätig (vermutlich das SK 10 a der Einsatzgruppe D, das seit Mitte August im Bereich der 11. Armee operierte.) Dieses führte „laufend Erhebungen durch, die zur Verhaftung und, wenn nötig, zur Beseitigung solcher Menschen führen, die sich während der Bolschewistenherrschaft an Leib und Gut der Volksgenossenschaften vergangen haben“. Es übernahm ferner - nach Maurers Bericht - den polizeilichen Schutz der Bevölkerung und stellte Personalausweise für "einwandfrei nachgewiesene Deutschblütigkeit“ aus. Die Förderung des deutschen Volkstums und seine Scheidung nach erwünschter und unerwünschter Substanz vollzogen sich also im wissentlichen Zusammenspiel der Kräfte - die „Aufbauarbeiten“ leistende Wehrmacht ließ durchaus Platz für die „Beseitigungen" durchführenden SS-Kommandos.

Neben Maurer nahm ein zweiter Volkstumsspezialist des DAI in den ersten Monaten des Rußlandfeldzugs in den Gemeinden der Deutschen in der UdSSR seine Tätigkeit auf, der ihm unterstellte Dienststellenleiter Dr. Karl Stumpp. Er war von seinem Landsmann Georg Leibbrandt, nun Leiter der Hauptabteilung I, Politik in Rosenbergs Ministerium, zum Leiter eines Sonderkommandos des RMO für Rußland bestimmt worden. Es war nicht zuletzt die Abkommandierung des SS-Sonderkommandos „R“ (Rußland) der Volksdeutschen Mittelstelle nach dem Osten, die Leibbrandt veranlaßt hatte, nun seinerseits ein Kommando zur Erfassung und Betreuung der Deutschen zu entsenden. Stumpp war von der Möglichkeit zum Einsatz in höchstem Maße angetan und setzte sich noch in der zweiten Julihälfte 1941, begleitet von einem zunächst kleinen Stab von Experten meist deutschrussischer Herkunft, mit einer Wagenkolonne in Bewegung. Allerdings war dieser Stab von Zivilisten, dem durch den irreführenden Namen Kommando Dr. Stumpp (seltener wurde der Name Sonderkommando Dr. Stumpp verwendet) der Anschein eines SD-Kommandos gegeben werden sollte, im rückwärtigen Heeresgebiet keinesfalls willkommen. Die SSKommandos schränkten seinen Aktionsradius in grober Willkür ein und zwangen Stumpp zu einer Reihe demütigender Kniefälle. Erst mit der Übergabe weiter Teile der östlichen Ukraine an die Zivilverwaltung wurde das bis dahin inoffiziell agierende Kommando Stumpp durch Erlaß des Reichsministers für die besetzten Ostgebiete vom 7.11. 1941 offiziell gebildet und der gesamte Stab von etwa 80 Personen mit Wagen und Schreibkräften nach Osten in Marsch gesetzt.

Stumpp, der während all der Jahre seiner Amtszeit im DAI nur auf den Tag des Einsatzes im befreiten Rußland gewartet hatte, war in Hochstimmung in das westliche Gebiet der Ukraine eingereist ${ }^{27}$. Im Gegensatz zu den Einheiten der Wehrmacht und den Einsatzgruppen, welche die deutschen Siedlungen sozusagen „unberührt“ angetroffen und sie noch im Operationsgebiet und weiter im rückwärtigen Heeresgebiet erfaßt hatten, fand das Kommando Stumpp die deutschen Dörfer nach einem ersten Prozeß der „Säuberung“ vor. Mit Feuereifer machte sich Stumpp nun in jedem deut-

27 Vgl. die sieben Erlebnisberichte Stumpps an seine Mitarbeiter im DAI ab Anfang August 1941, GR T 81, R 599, F 5386517-64. 
schen Dorf auf seinem Weg von Schitomir bis Dnjepropetrowsk (früher Jekaterinoslaw) an die Arbeit: Überall stellte er detaillierte „Ortsberichte" zusammen, in denen er die Geschichte der deutschen Siedlungen von der Oktoberrevolution bis zum deutschen Einfall nach Auskünften der Ortsansässigen rekonstruierte. Diese Berichte ${ }^{28}$ enthalten genaueste Angaben über die Verluste im Ersten Weltkrieg, im Bürgerkrieg, in den Hungersnöten, die Zeiten der Entkulakisierung und des politischen Terrors. Sie beschreiben die innere, soziale und demographische Struktur der Siedlungen, ihre sozialen und schulischen Einrichtungen und vermitteln ein präzises Bild von der Lage der deutschen Dörfer nach der „Befreiung“ durch die Wehrmacht. Auf diesen Dorfund Ortsberichten basierten die zusammenfassenden Berichte für die einzelnen Siedlungsgebiete, die Stumpp vor Ort zusammenstellte und an seine Dienststellen, das DAI und das RMO, sandte ${ }^{29}$. Den Berichten fügte Stumpp, der sich seit Ende der dreißiger Jahre zunehmend auf die volks- und rassenbiologische Erforschung des Rußlanddeutschtums spezialisiert hatte ${ }^{30}$, auch exakte Daten über „völkische Mischfälle“ in den deutschen Gemeinden, „Mischehen“, „Mischlinge“ sowie „fremdvölkisch gemischte" Familien bei.

Diese einem historisierenden und statistischen Übereifer entsprungenen Erzeugnisse mochten dann in vielen Fällen einen Leitfaden für die die deutschen Gebiete „säubernden" SS-Kommandos abgeben.

Nach der Zusammenstellung der Ortsberichte nahm Stumpp eine umfassendere Aufgabe in Angriff: die Arbeiten zur Gründung eines „Zentralsippenamtes“ der Rußlanddeutschen in der besetzten Sowjetunion ${ }^{31}$. Stumpp hatte bereits für die Ortsberichte „sippenkundliche Fragebögen“ ausfüllen lassen und die örtlichen Register (Kirchenbücher und -matrikeln, Trau-, Geburts- und Sterberegister) „sichergestellt“. Diese Materialien sollten den Grundstock einer zentralen Sippenkartei aller deutschen Familien bilden, mit deren Hilfe sich strittige Fälle lösen lassen würden. Dem Zentralsippenamt sollten folgende Aufgaben zufallen:

„1. Die Ausstellung und Beschaffung von Urkunden und Gutachten für den Nachweis der arischen Abstammung und die Aufstellung von Stammbäumen und Ahnentafeln. Außerdem hat das Zentralsippenamt laufend die volksbiologische Lage und Weiterentwicklung zu erforschen und zu verfolgen.

2. Zusammentragung und Verwaltung der gesamten Kirchenmatrikel und des sippenkundlichen Materials ...

3. Auswertung alles sonstigen Materials zur Wiederherstellung der verlorengegange-

${ }^{28}$ LC-DAI, Metal edge boxes 146-154 enthalten die Originale der Ortsberichte.

${ }^{29}$ Vgl. u. a. Bericht über die 19 deutschen Siedlungsgruppen des Chortitza-Gebietes auf der Westseite des Dnjepr, Generalbezirk Dnjepropetrowsk, LC-DAI Box 146; Zusammenfassender Bericht über die deutschen Siedlungen im Gebiet Korosten, LC-DAI, Box 151; Zusammenfassender Bericht über die deutschen Siedlungen im ehemaligen Wolhynien, d.h. den heutigen Generalbezirken Shitomir, Rowno und Luzk, einschließlich Uman, das zu Kiew zählt, LC-DAI, Box 152.

${ }^{30} \mathrm{Vgl}$. die Arbeit von Stumpp, K., Die Volksbiologie der Rußlanddeutschen (1940), GR T 120, R 1496, F 626197-200.

${ }^{31}$ Vgl. Kommando Dr. Stumpp, Berlin, den 17. November 1942, An das Reichsministerium für die besetzten Ostgebiete, Betr.: Zentralsippenamt und Unterabteilungen, LC-DAI, Box 154. 
nen Kirchenmatrikel (Sippenkundliche Fragebögen des Kommandos Dr. Stumpp, Kauf- und Pachtverträge und sonstiges Gerichtsaktenmaterial, Seelen- und Volkszählungslisten, Bibeleintragungen, Grabdenkmalsinschriften usw.).

4. Das Sippenamt muß wegen der in den letzten 20 Jahren eingegangenen Mischehen und der in der Gründungszeit in katholischen und orthodoxen Kirchen vollzogenen Taufen und Trauungen auch über das gesamte Nichtdeutsche Matrikel- und Archivmaterial verfügen dürfen.

5. Nach dem Kriege ist mit einer Rückwanderung eines Teils der in Deutschland, in der Verbannung und Übersee zerstreuten Rußlanddeutschen zu rechnen. Für diese wird es äußerst schwierig, wenn nicht unmöglich sein, sich Urkunden zu verschaffen, da ihre ehemaligen Heimatdörfer aufgelöst und umgesiedelt wurden und die Kirchenmatrikel für sie unauffindbar sind. Aus all diesen Gründen ist die Verzettelung des Materials und der Arbeitskräfte nicht zu verantworten, sondern die Gründung eines Zentralsippenamtes ein unbedingtes Erfordernis.“

Stumpps Anregungen fielen im Reichsministerium Ost auf fruchtbaren Boden. Allerdings wurden anstelle eines zentralen Sippenamtes mit Sitz in Dnjepropetrowsk und Unterabteilungen in den Hauptstädten der Generalkommissariate lediglich zwei Sippenämter bei den Generalkommissaren von Schitomir und Dnjepropetrowsk geschaf$\mathrm{fen}^{32}$. Ihr Auftrag bestand darin, die sippenkundlichen Materialien und Archivalien für das „Deutschtum im Reichskommissariat Ukraine“ zusammenzufassen und auszuwerten; der Aufgabenbereich überschritt also nicht die Grenzen der unter deutscher Zivilverwaltung stehenden Gebiete. Auf der Grundlage des gesammelten Materials sollten „Abstammungsnachweise“ geführt und „Abstammungsurkunden“ oder in fraglichen Fällen „Abstammungsgutachten“ ausgestellt werden. Rassenpolitische Arbeit im engeren Sinne leisteten die Sippenämter nicht ${ }^{33}$. Das Sippenamt im Generalkommissariat Schitomir war für die westliche und Zentralukraine (die Bezirke Lutzk, Schitomir, Kiew bis einschließlich Tschernigow) und das Sippenamt beim Generalkommissar Dnjepropetrowsk für die östlichen, zentralen und südlichen Teile des Reichskommissariats Ukraine (die Generalbezirke Dnjepropetrowsk, Nikolajew, Stalino und Charkow, einschließlich der unter Militärverwaltung stehenden Krim) zuständig. „Die Sippenämter gehören zum Dienstgebrauch der Hauptabteilungen Politik der Generalkommissare in Schitomir und Dnjepropetrowsk", d.h. sie unterstanden also direkt der von Leibbrandt geführten Hauptabteilung Politik im $\mathrm{RMO}^{34}$. Die Durchführungsbestimmungen ${ }^{35}$ nahmen wörtlich die Formulierungen aus

${ }^{32}$ Runderlaß des RMO vom 17. Dezember 1942 - II c 2440.

${ }^{33}$ Vgl. Reichsministerium für die besetzten Ostgebiete, Hauptabteilung I, Ie, Berlin, März 1943, GR T 454, R 105, F 1099-1108, hier 1106: „Rassenkundliche und rassenpolitische Arbeit kann und will das Sippenamt nicht leisten."

${ }^{34}$ Abschrift von Abschrift. Errichtung von Sippenämtern bei den Generalkommissaren in Shitomir und Dnjepropetrowsk. Zbl. RKU 1942, LC-DAI, Box 154.

${ }^{35}$ Durchführungsbestimmungen zum Runderlaß vom 17.12. 1942 - II c 2440 - über Errichtung von Sippenämtern bei den Generalkommissaren in Shitomir und Dnjepropetrowsk, Runderlaß vom 7. Januar 1943 - II a/2, LC-DAI, Box 154. 
Stumpps Plan zur Errichtung der Sippenämter auf; sie unterstrichen die Bedeutung der „Ausstellung und Beschaffung von Urkunden und Gutachten für den Nachweis der arischen Abstammung" und betonten die Notwendigkeit einer fortlaufenden „volksbiologischen“ Überwachung des Rußlanddeutschtums. Sie forderten, daß die „bisher von der Volksdeutschen Mittelstelle, vom Kommando Dr. Stumpp oder von anderen Dienststellen gesammelten Urkunden oder sonstigen Unterlagen" sofort an die in Gründung befindlichen Sippenämter weitergeleitet wurden, ein Verlangen, dem allein das Kommando Dr. Stumpp nachkam. Die Sippenämter wurden ermächtigt, „aufgrund von Unterlagen und eidesstattlichen Erklärungen von Zeugen rechtsgültige Urkunden gegen Gebühr auszustellen. Die Volksdeutschen im Reichskommissariat Ukraine und die aus dem Reichskommissariat Ukraine zum Arbeitseinsatz im Reich weilenden Volksdeutschen sind von der Gebührenleistung befreit."

Wie weit die Arbeit dieser Sippenämter noch zum Tragen kam, läßt sich aus dem überlieferten Material nicht ersehen. In einigen Fällen ersuchten die Mitarbeiter der Einwandererzentrale die Sippenämter um Gutachten über die Rassenreinheit der im Prozeß der „Durchschleusung" befindlichen Deutschen aus Rußland. So mußten Stumpp und seine Mitarbeiter z.B. Rassengutachten für den als nichtarisch verdächtigten Pius Eckstein aus Simonsfeld, Rayon Apostolowo, Generalbezirk Dnjepropetrowsk, ausstellen. Auch die Anträge auf Zuerkennung der deutschen Volkszugehörigkeit eines Isaack und eines Lachmann liefen durch Stumpps Hände. In einzelnen Fällen mochten besonders die alttestamentarischen Namen der Mennoniten deutscher und holländischer Herkunft ihre „Rassenmusterung " in der Einwandererzentrale erschweren.

Stumpp selbst blieb für seine sippenkundliche Arbeit wenig Zeit. Nach der Wende des Kriegs im Winter 1942/43 mußte das Stumpp unterstehende Sippenamt Dnjepropetrowsk, zusammen mit der Dienststelle seines Kommandos, geräumt werden. Das Sippenamt wurde nach Schitomir verlegt ${ }^{36}$, das Kommando Dr. Stumpp aufgelöst ${ }^{37}$. Stumpp selbst verließ die Dienststelle am 21.2. 1943. Die Archivalien, in Sondertransporten an Leibbrandt geschickt, gingen zum großen Teil verloren. Mit der Entfernung Leibbrandts aus seinem Amt (Sommer 1943) verlor Stumpp seinen Patron und Fürsprecher.

Die letzte Phase des Einsatzes von Stumpp, der ab Herbst 1943 zur Betreuung der in der Westukraine aufgefangenen deutschen Flüchtlinge abkommandiert war, kenn-

${ }^{36}$ Außendienststelle Dr. Stumpp, Dnjepropetrowsk, den 27. Februar 1943. An den Herrn Reichsminister für die besetzten Ostgebiete, Betr.: Verlegung der Außendienststelle Dnjepropetrowsk nach Shitomir, LC-DAI, Box 154.

37 Vgl. Schreiben des Reichskommissars für die Ukraine, II a - 2, Rowno, den 23. Dezember 1942, LC-DAI, Box 154; am 22.2. 1943 fand im RMO eine Besprechung zwischen Leibbrandt, Kinkelin, Maurer und Stumpp statt, auf der der Beschluß zur Auflösung des Kommandos Dr. Stumpp gefaßt wurde (vgl. Der Reichsminister für die besetzten Ostgebiete, Nr.Ia - 193, Berlin, den 22.2. 1943. Schnellbrief! An den Reichskommissar für die Ukraine, Rowno. Betr.: Abschluß der Arbeiten des Kommandos Dr. Stumpp, LC-DAI, Box 154); Schreiben Dr. Stumpp, An den Reichsminister für die besetzten Ostgebiete, Abt. I, 1 c, Shitomir, den 4.3. 1943, LC-DAI, Box 154. 
zeichneten Verzweiflung, Depression und Isolation. Sein Briefwechsel, besonders mit seinem Landsmann und Mitarbeiter Eduard Krause, verdeutlicht die Desillusionierung des Deutschrussen, der sich selbst in zunehmendem Maß als Opfer der Maschinerie der nationalsozialistischen Stäbe empfand ${ }^{38}$.

\section{Die Einsatzgruppen der Sicherheitspolizei und des SD}

Die für den Einsatz auf sowjetischem Territorium bestimmten Einsatzgruppen der Sicherheitspolizei und des SD waren etwa drei Wochen vor Beginn des Rußlandfeldzuges zusammengestellt worden ${ }^{39}$. Während die Leiter der Einsatzgruppen und mit ihnen das Gros der Leiter und Mannschaften der Einsatz- und Sonderkommandos gebürtige Reichsdeutsche waren, befanden sich in den Rängen der Einsatz- und Sonderkommandos auch in Rußland geborene Deutsche ${ }^{40}$. Sie waren entweder in den zwanziger und dreißiger Jahren aus der Sowjetunion geflohen ${ }^{41}$ bzw. ausgebürgert worden ${ }^{42}$ oder aber 1939/40 im Zuge der Vertragsumsiedlung aus der sowjetischen Interessensphäre (den baltischen Provinzen, Bessarabien, Wolhynien usw.) in das nationalsozialistische Deutschland gelangt. Vor allem Deutsche aus Wolhynien waren in größerer Zahl, u.a. als Dolmetscher und NSKK-Leute, in den Sonder- und Einsatzkommandos tätig. Auch Volksdeutsche aus Polen waren im Einsatz.

Von den vier auf sowjetischem Boden operierenden Einsatzgruppen (Einsatzgruppen A, B, C, D) traf die meisten Rußlanddeutschen die Einsatzgruppe D (Chef: Otto Ohlendorf) an, deren Weg durch das gesamte Schwarzmeergebiet bis hin zum Kaukasus

${ }^{38}$ Siehe u.a. GR T 81, R 636, F 5437341-51.

${ }^{39}$ Vgl. Krausnick, H., Wilhelm, H.-H., Die Truppe des Weltanschauungskrieges. Die Einsatzgruppen der Sicherheitspolizei und des SD 1938-1942. Teil 1. Krausnick, H., Die Einsatzgruppen vom Anschluß Österreichs bis zum Feldzug gegen die Sowjetunion. Entwicklung und Verhältnis zur Wehrmacht, Stuttgart 1981. 23 Führer und Verantwortliche der Einsatzgruppen wurden in Nürnberg (Case 9) für ihre Verbrechen abgeurteilt; vgl. Trials of War Criminal before the Nuremberg Military Tribunal (TWC), Bd.IV, „The Einsatzgruppen Case“, „The RuSHA Case“, Nürnberg Okt. 1946-April 1949.

${ }^{40}$ Nach heutigem Kenntnisstand kann die Bemerkung Reitlingers, die Mitglieder der Einsatzkommandos seien "mostly Volksdeutsche" gewesen, nicht mehr als zutreffend betrachtet werden (Reitlinger, G., The SS. Alibi of a Nation, 1922-1945, Melbourne 1956, S. 180.).

${ }^{41}$ So u.a. W.G.K., 1912 in der deutschen Kolonie Helenendorf im Kaukasus geboren, hatte die Enteignung und Verbannung seines Vaters, eines wohlhabenden Kolonisten, miterlebt. Er war 1928 über Persien nach Deutschland (1930) geflohen. 1937 Beitritt zur NSDAP und Mitglied der Gestapo. 1940 Dolmetscher bei der deutsch-sowjetischen Umsiedlungskommission. Anfang Juni 1941 Abordnung ins Ausbildungslager Düben der Einsatzgruppe D. Notorischer Juden- und Russenhasser. Wegen 5000 nachgewiesener (!) eigenhändiger Tötungen (Einzeltötungen, Erschießungen jüdischer Gefangener, Tötungen durch Gaswagen, Tötungen verbunden mit „unnötigen “ Mißhandlungen etc.) Anfang der 70er Jahre in Deutschland vor Gericht gestellt. Massives Anklagematerial aus der UdSSR, die K. Anfang der 60er Jahre trotz seines geänderten Namens ortete und identifizierte (Lev Ginzburg schrieb einige erhellende Artikel zu K. in der Litaraturnaja Gazeta, 1963).

${ }^{42}$ U.a. A.L., einer alten deutschen Familie Moskaus entstammend, 1906 in Moskau geboren, seit 1934 deutscher Staatsbürger, Mitglied der Einsatzgruppe D, Durchführung eigenhändiger Gewaltverbrechen. 
führte. Von ihr wurden im südrussischen Raum die auf und südlich der Linie Tschernowitz, Mogilew-Podolskij, Jampol, Ananew, Nikolajew, Melitopol, Mariupol, Taganrog, Rostow, der Krim und dem Kauskasus gelegenen deutschen Siedlungen erfaßt. Den nördlichen und zentralen Teil der Ukraine „durchkämmte“ die Einsatzgruppe $C$ (Chef: Dr. Rasch). Für die weißrussischen Gebiete waren die Einsatzgruppen A und B zuständig, das besetzte Gebiet im Norden der Sowjetunion (z. B. die deutschen Siedlungen um Leningrad und Schlüsselburg) war alleiniger Kontrollbereich der Einsatzgruppe A.

Die Einsatzgruppen bestanden jeweils aus etwa 500 bis 800 Mann. Sie führten ihre Aufgaben in unmittelbarer Nähe und Zusammenarbeit mit den Formationen der Wehrmacht in den Operationsgebieten der Front aus. Im Chaos der vorrollenden Armee sollten sie auf Anweisung ihres Dienstherrn Heydrich vom 2.7. 1941 die „sicherheitspolizeiliche Befriedung" der neu besetzten Gebiete mit rücksichtsloser Schärfe und auf breitestmöglicher Basis durchführen. Heydrichs Weisung lautete im einzelnen:

„Zu exekutieren sind alle

Funktionäre der Komintern (wie überhaupt alle kommunistischen Berufspolitiker schlechthin),

die höheren, mittleren und radikalen unteren Funktionäre der Partei, der ZK's, der Gau- und Gebietskomitees,

Volkskommissare,

Juden ...

sonstige radikalen Elemente (Saboteure, Propagandeure, Heckenschützen, Attentäter, Hetzer usw.). ${ }^{{ }_{43}}$

Die Befehle zur Ausrottung unerwünschter „Fremdstämmiger“ (Juden, Slawen, Zigeuner) und politisch verdächtiger Elemente, unter ihnen auch Volksdeutsche, gingen von Himmler aus und wurden mündlich ${ }^{44}$ über Heydrich und seinen Adjutanten und späteren Nachfolger Kaltenbrunner an die Führer der Einsatzgruppen übermittelt. Diese gaben sie ebenfalls in der Regel mündlich an die Leiter der Einsatz- und Sonderkommandos weiter.

Wenige Wochen nach Beginn des Unternehmens Barbarossa beschloß Himmler, vermutlich auf Drängen des Leiters der Volksdeutschen Mittelstelle in Berlin, Werner Lorenz, die Tätigkeit der Einsatzgruppen mit der der Volksdeutschen Mittelstelle zu verbinden und zu koordinieren: Hand in Hand mit der Vernichtung fremden Volkstums sollte die Festigung eigenen Volkstums durchgeführt werden. In seinem Schreiben vom 11.Juli 1941 an Lorenz als Chef der Vomi und Heydrich als Chef der Einsatzgruppen beauftragte Himmler „die Volksdeutsche Mittelstelle, alle Maßnahmen zu treffen, um das Volksdeutschtum in der besetzten Sowjetunion zu erfassen und durch Aufstellung nichtbolschewistischer Vertrauensmänner den Grundstein zu einer

\footnotetext{
${ }^{43}$ Landgericht München 1, Urteil in der Strafsache gegen S., W., E. der Einsatzgruppe D, Einsatzkommando 11b, Az.: 115 Ks 6/71-7, S. $15 \mathrm{ff}$.

${ }^{44} \mathrm{Vgl}$. Reitlinger, SS, S. 180.
} 
deutschen Führung zu legen ... Die Arbeit hat im engsten Zusammenschluß und Einvernehmen mit den Einsatzkommandos der Sicherheitspolizei zu erfolgen ${ }^{* 45}$. Himmler unterstellte die Beauftragten der Vomi den Höheren SS- und Polizeiführern in den entsprechenden Gebieten der Heeresgruppen „und bei diesen den Befehlshabern der Sicherheitspolizei. Die Höheren SS- und Polizeiführer sowie die Befehlshaber der Sicherheitspolizei haben diese Tätigkeit mit allen Kräften zu fördern." Darüber hinaus erteilte Himmler „dem Höheren SS- und Polizeiführer, in dessen Gebiet das Wolgadeutschtum fällt", nämlich SS-Standartenführer Guntram Pflaum ${ }^{46}$, einen „Sonderauftrag Rußland“; Pflaum, hauptamtlicher Verantwortlicher für den „Lebensborn" im Persönlichen Stab des Reichsführers SS, sollte sich im Wolgagebiet „nach Lage der örtlichen Verhältnisse sofort der noch blutlich guten und unvermischten Kinder" annehmen. Dieser gab sich mit der unsicheren Ausbeute der rassisch zweifelhaften deutschen Kinder im Wolgagebiet nicht zufrieden. In einem persönlichen Vortrag im Führerhauptquartier am 16. August 1941 verstand er es durchzusetzen, daß sein Aufgabengebiet „auf die gesamten besetzten Gebiete der UdSSR“ ausgeweitet wurde ${ }^{47}$.

Die Anordnung Himmlers hatte zur Folge, daß der Aufgabenbereich jener Einsatzkommandos, welche die Erfassung der Volksdeutschen in den ersten Wochen durchführten, etwa ab Mitte August sukzessive auf die SS-Kommandos der Vomi überging. Zwischen diesen, in erster Linie dem SS-Sonderkommando „ $R$ “ unter der Leitung von SS-Brigadeführer und General der Polizei Horst Hoffmeyer und seinen Untergruppierungen, und den Einsatzgruppen herrschten bestes Einvernehmen und enge Zusammenarbeit ${ }^{48}$. Der Chef der Einsatzgruppe D, Ohlendorf, übergab noch im August 1941 den Sektor „Betreuung“ der Volksdeutschen im rumänisch besetzten Teil des Schwarzmeergebietes an Hoffmeyer. In seiner Eigenschaft als Leiter des SSSonderkommandos „R“ der Vomi unterstand Hoffmeyer sowohl Lorenz, dem Leiter der Vomi in Berlin, als auch Prützmann, dem Höheren SS- und Polizeiführer Rußland Süd.

$\mathrm{Zu}$ Anfang der Erfassung der Deutschen in den frisch erkämpften Frontabschnitten machten die Einsatzgruppen eine Reihe unerwarteter Beobachtungen. Die erste betraf das Ausmaß der sowjetischen Zwangsaussiedlung der Deutschen, die unmittelbar nach dem deutschen Angriff auf die Sowjetunion eingesetzt hatte. Die im deutschen Blitzvormarsch eroberten Gebiete Wolhyniens, Podoliens und der westlichen Ukraine waren noch von der Totalaussiedlung verschont geblieben. Auch die Siedlungen des Schwarzmeergebietes bis hin zum Bug waren aufgrund des raschen deutsch-

${ }^{45}$ Der Reichsführer-SS, Az/363 a/3, Führer-Hauptquartier, den 11. Juli 1941, Betr.: Erfassung der deutschen Volkszugehörigen in den Gebieten der europäischen UdSSR, Nbg. Dok. NO-4274.

46 SS-Standartenführer Guntram Pflaum, SS-Nr. 39477, Parteieintritt Mai 1932.

${ }^{47}$ Schreiben Pflaums, Berlin, den 25. August 1941, Nbg. Dok. NO-4273.

${ }^{48} \mathrm{Vgl}$. Affidavit of Otto Ohlendorf, 9 Dec. 1947, concerning the tasks of the Einsatzgruppen with respect to Ethnic Germans in the USSR, Lorenz Document 51, Lorenz Defense Exhibit 26, TWC, IV, S. $853 \mathrm{f}$. Als Beweis für die enge Zusammenarbeit zwischen Einsatzgruppe B und Vomi im Gebiet der Heeresgruppe Mitte vgl. Nbg. Dok. NO-5095, 6. Juli 1942. 
rumänischen Vorstoßes der Evakuierung entgangen. Je weiter östlich jedoch die Einsatzgruppen ihre Erfassungsarbeiten durchführten, desto geringer wurde die deutsche Bevölkerungsdichte; jenseits des hartumkämpften Dnjepr waren nur noch vereinzelt geschlossene Siedlungen erhalten. Die überwiegende Mehrzahl der deutschen Dörfer war gänzlich geleert worden. Im gesamten Gebiet östlich des Dnjepr existierte nur noch „Streudeutschtum"; mehr oder minder geschlossene Ortschaften in den Gebieten von Don und Kuban, von Terek und Kaukasus, auf der Krim und am Asowschen Meer waren nun „erloschen“.

Die sowjetische Zwangsaussiedlung der Bevölkerung deutscher Nationalität war in den Sommermonaten 1941 nach Listen erfolgt, welche auf Anordnung des Zentralkomitees der KPdSU bereits im Herbst 1934 angelegt worden waren. Die Aussiedlung vollzog sich in drei Stufen. Zuerst wurde die männliche Bevölkerung im Alter von ca. 16-60 Jahren mit dem Vieh, den Gerätschaften und den Maschinen der Siedlungen in Richtung Osten in Marsch gesetzt. Volksdeutsche Männer, die als Sympathisanten für Deutschland gelten konnten, wurden meist noch im Ort erschossen (ebenso kollaborationsverdächtige Ukrainer und Weißrussen) ${ }^{49}$. In der zweiten Stufe der Zwangsaussiedlung wurden die deutschen Frauen und Mädchen aus den Orten zum Bau militärischer Anlagen (Schanzen, Panzergräben, Wälle etc.) abgezogen, die Zurückgebliebenen hatten die Ernte einzubringen. Der letzte Schritt, die totale Aussiedlung, führte die deutsche Restbevölkerung unter Einsatz aller verfügbaren Transportmittel (Lastautos bis Güterzüge) in einem qualvollen vielmonatigen Weg in die Gebiete jenseits des Ural. Ca. 650000 Deutsche wurden auf diese Weise aus den europäischen Teilen der UdSSR aus- und in den asiatischen Gebieten angesiedelt.

Die zweite Überraschung für die Einsatzkommandos war, daß selbst in den Gebieten, die von der sowjetischen Zwangsaussiedlung ganz oder partiell verschont geblieben waren, der Bevölkerungsaufbau der deutschen Orte starke Anomalien aufwies. Infolge der Wellen von Verhaftungen, Deportationen und Verschleppungen war der Anteil der männlichen Bevölkerung auf die Hälfte der weiblichen abgesunken; Kinder unter vierzehn Jahren machten einen überdurchschnittlich hohen Anteil der Gesamtbevölkerung aus.

Als dritte Beobachtung kam hinzu, daß sich das Erscheinungsbild der Volksdeutschen, die in den westsowjetischen Gebieten und im Schwarzmeerraum einen relativ gesunden und "ungebrochenen" Eindruck machten, in Richtung Osten und Norden der UdSSR zunehmend verschlechterte.

Die Angehörigen der Sicherheitspolizei und des SD waren vor ihrem Einsatz durch die Arbeiten der Deutschtumsabteilungen der Antikomintern und des Amtes Osten der NSDAP auf ihre Tätigkeit vorbereitet worden. Beim Kontakt und Umgang mit der vorgefundenen deutschstämmigen Bevölkerung erkannten sie sehr bald, daß die militärischen und sicherheitspolitischen Vorberichte der Abteilungen grundsätzlich falsch waren ${ }^{50}$. So kamen ihnen die Deutschen der „befreiten“ Gebiete durchaus nicht

\footnotetext{
${ }^{49}$ Vgl. Ereignismeldung UdSSR Nr. 24, 16. Juli 1941, S. 10 und Nr.13, 5. Juli 1941, S. 3.

so Ereignismeldung UdSSR Nr.12, 4.7.1941.
} 
einhellig mit der dankbaren Begeisterung entgegen, wie sie von den deutschrussischen Emigranten jener Dienststellen vorausgesagt worden war. Die ersten im Gebiet von Nowograd-Wolynsk (Zwiahel) angetroffenen Deutschen verhielten sich "freundlich, aber reserviert" ${ }^{\text {"51 }}$. Hingegen versprachen die als „Hilfspolizeibeamte“ (d.i. genauer: Hilfsfreiwillige oder Hiwis) eingesetzten ersten Überläufer aus den früheren taurischen und Wolga-Kolonien, daß im Innern der UdSSR „die Deutschen, mit Ausnahme weniger Kommunisten, die Befreiung freudig begrüßen werden“. Das äußere Bild der deutschen Siedlungen in der Westukraine war - laut Einsatzgruppenberichten - überraschend gut ${ }^{52}$. Zwar zeigte die deutsche Bevölkerung charakteristische, durch ihre Geschichte bedingte Deformationen, wie niedrigen Bildungsstand, Schwund der Intelligenzschicht und Dominieren der niedrigen Berufsgruppen bzw. Beruflosen ${ }^{53}$; doch war die Haltung dieser Menschen „ungebrochen“: Bolschewisierung hatte nicht stattgefunden; die Kinder sprachen deutsch. „Der rassische und charakterliche Wert dieser Menschen ist als gut anzusehen". Biologisch ungebrochen erschienen den Einsatzkommandos der Einsatzgruppe D auch die Deutschen der Ostukraine ${ }^{54}$. Die deutschen Siedlungen um Kriwoj Rog, gemischte Siedlungen von Katholiken, Lutheranern und Mennoniten, in denen meist ein älterer Bauer als Prediger gemischte Gottesdienste leitete, zeichneten sich durch Sauberkeit und soliden Hausbau (Steinhäuser mit Ziegeldächern) aus. Die Menschen zeigten eine durchaus negative Einstellung zu Kommunismus und Sowjetsystem. Sie hatten zum großen Teil „das Deutschtum reinerhalten“. Nach Ansicht der Berichterstatter stellte dieser ,harte arbeitssame Schlag" mit seiner großen biologischen Vitalität „ein unentbehrliches Element" für die zukünftige extensive Bewirtschaftung der Ukraine dar. Nur in der Generation der, meist vaterlosen, Halbwüchsigen wurden ungehemmte Aufweichungstendenzen des Deutschtums konstatiert.

Nach Erfassung eines Drittels der deutschen Siedlungen der Ukraine gelangte die Einsatzgruppe $\mathrm{C}$ zu einem ersten abschließenden Urteil ${ }^{55}$. Sie sah die Befürchtung, die Deutschen der UdSSR „bolschewisiert" zu finden, als „völlig unbegründet“ an: „Ist es schon den Bolschewisten nicht gelungen, die ukrainische Landbevölkerung für sich zu gewinnen, so stieß sie bei den Deutschen auf äußeren und inneren Widerstand, der mit brutalstem Terror gebrochen wurde. Das Deutschtum hat außerordentliche Verluste erlitten, ist äußerlich vielleicht verwahrlost und in einigen Gebieten führerlos - bolschewisiert ist es jedoch nicht ... Gesamteindruck: wirtschaftlich sehr tüchtig. Trotz einer gewissen Schwächung seine Leistungskraft und Initiative nicht erloschen; das gesamte dörfliche Leben gut organisiert. Keinerlei völkische oder weltanschauliche Fremdeinflüsse." In den meisten Gemeinden habe die religiöse Einstellung und der Familiensinn das Eindringen kommunistischer Gedankengänge verhindert. „Ernste biologische Gefahren“ sahen die Einsatzgruppen an den Orten, in de-

\footnotetext{
${ }^{51}$ Ereignismeldung UdSSR Nr. 26, 18. Juli 1941.

52 Ereignismeldung UdSSR Nr. 81, 12.9. 1941, S. $19 \mathrm{ff}$.

${ }^{53}$ Ereignismeldung UdSSR Nr.75, 6.9. 1941, S. 4.

${ }^{54}$ Ereignismeldung UdSSR Nr. 85, 16.9. 1941, S. $16 \mathrm{ff}$.

${ }^{55}$ Ereignismeldung UdSSR Nr. 86, 17.9. 1941, S. 29-35.
} 
nen die deutschen Männer fehlten. Hier hätten sich deutsche Frauen und Mädchen oft mit Ukrainern, Russen und sogar Juden „eingelassen“. Die Zahl der unehelichen Kinder mit „fremdvölkischem Einschlag" liege in diesen Siedlungen bei 9-10\%.

Östlich der Dnjepr-Linie änderte sich das Bild der erfaßten Deutschen stark. Die Deutschen, die sich nur sehr zögernd auf die Maueranschläge hin meldeten und registrieren ließen, erschienen den Einsatzkommandos „verängstigt“, „reserviert“ bis „sehr reserviert" und „wenig entgegenkommend“. Diese Haltung wurde als Furcht vor sowjetischen Repressalien nach einem möglichen deutschen Abzug interpretiert eine Erklärung, die sicher nicht das ganze Spektrum der Gefühle dieser Menschen abdeckte. Denn mittlerweile war auch in diesen Teilen der Sowjetunion bekannt geworden, was die sowjetische Bevölkerung von den Einsatzgruppen zu erwarten hatte. Die Bewertung des Streudeutschtums in den Gebieten östlich des Dnjeprs war nicht sehr günstig. Das äußere Erscheinungsbild dieser Menschen wurde als wenig befriedigend, ihre Bindung an das deutsche Volkstum als wenig eng bezeichnet. Die Assimilation an die andersethnische Umgebung, besonders an das Russentum, sei weit fortgeschritten, Deutschkenntnisse und deutsche Erziehung in der jüngeren Generation seien kaum noch festzustellen.

Noch schlechter fiel die Bewertung der Deutschen in den ukrainischen Großstädten aus. Nach Berichten der Einsatzgruppe C (Standort Kiew) ${ }^{56}$ war das „volksdeutsche Bewußtsein" der deutschen Stadtbevölkerung in der Ukraine „fast völlig verschüttet ... Die Zahl der Mischehen ist außerordentlich hoch. Die deutsche Sprache wird nur noch teilweise gesprochen. Die meisten Kinder haben sie überhaupt nicht mehr gelernt. In den Industriegebieten des Donezgebietes kommt noch hinzu, daß ein erheblicher Teil Volksdeutscher bolschewisiert ist."

Nach vielmonatigen Beobachtungen wurden aber auch die Wertungen der Deutschen in geschlossenen Siedlungen negativer. So meldeten dieselben Berichte, daß von den vorwiegend deutschen Siedlungen sich nur die Mennonitenkolonien „völkisch rein“ erhalten hätten. „In den weiteren Siedlungen ging das Deutschtum durch Mischehen mit russischer und ukrainischer Bevölkerung mehr oder minder verloren." Selbst die Befunde des zunächst recht enthusiastisch beurteilten Schwarzmeerdeutschtums wurden bei längerer Beobachtung nach unten korrigiert. Die Einsatzgruppe D berichtete Anfang Oktober 1941, daß die deutsche Bevölkerung der Dörfer im Berezaner Gebiet, nun zum rumänisch verwalteten Transnistrien gehörend, aufgrund von Arbeitsunlust und dem Verlust des Gefühls für den ethischen Wert der Arbeit besonders in der mittleren und jüngeren Generation „erheblich an Wert eingebüßt" habe ${ }^{57}$. Verschwiegen wurde dabei, daß sich die Hoffnungen der örtlichen Bevölkerung auf die Auflösung der Kollektive und auf eine Verminderung des harten Arbeitsdrucks und der hohen Abgaben nicht erfüllt hatten und daher eine Form passiven Widerstands bestand. Der Bericht sprach weiter von einem weithin wahrnehmbaren Zerfall der moralischen Haltung: Deutsche Frauen gingen „sittlich zugrunde ...,

${ }^{56}$ Ereignismeldung UdSSR Nr.187, 30.3. 1942, S.9f.

57 Ereignismeldung UdSSR Nr. 103, 4.10. 1941, S.5-9. 
indem sie sich mit Bauern ihres Kollektivs einließen“; die jüngere Generation zeigte „Anfänge des volkstumsmäßigen Vermischungsprozesses mit Russen, Ukrainern und sogar Juden“. Um weitere „blutmäßige Verbindungen mit Andersrassischen nicht zu einer Allgemeinerscheinung" werden zu lassen, forderte die Einsatzgruppe D die baldige Anwendung der Deutschen Volksliste und damit die Scheidung der Gemeinden und Familien nach „verwendbarem und unbrauchbarem Blut- und Rassebestand“.

Besondere Probleme bereitete den Einsatzkommandos immer wieder die Religiosität der Deutschen in der UdSSR: „Fast alle Volksdeutschen sind ausgesprochen christlich eingestellt. Ihre kirchliche Bindung hat zum Teil sektiererischen Charakter." ${ }^{\text {"58 }}$ Doch wußte man die Tatsache zu schätzen, daß der Kampf der Sowjetregierung gegen die Religion - hier enthüllt sich die Symmetrie der Systeme - die in den deutschen Dörfern zuvor bestehende scharfe Trennung nach Konfessionen gelockert und zum Teil aufgehoben hatte. Damit seien erste Ansätze zu einem „volksgemeinschaftlichen Denken“ im Sinne des Nationalsozialismus möglich geworden ${ }^{59}$. Klagen wurden allerdings darüber geführt, daß in den deutschen Siedlungen des Schwarzmeergebietes der „Konfessionalismus" das „Deutschtumsbewußtsein“ immer noch überschreite. Z.B. seien in den Berezaner Siedlungen religiöse Gegensätze zwischen den Deutschen oft stärker ausgeprägt gewesen als „die Volkstumsunterschiede zwischen Volksdeutschen und den sie umgebenden fremdvölkischen Gruppen ... So fühlten die Katholiken sich eher zu den ihnen glaubensmäßig näherstehenden russisch-orthodoxen Russen hingezogen" als zu den protestantischen. Allerdings habe auch hier die Herrschaft des kommunistischen Atheismus die Grundlagen zu einer Entkonfessionalisierung gelegt, auf die die nationalsozialistische Erziehung aufbauen könne ${ }^{60}$. Gemäß der Anweisung Hitlers in der Planungssitzung vom 16.7. 1941, „die Tätigkeit von Kirchen käme keinesfalls in Frage ${ }^{c_{61}}$, vertraten die Einsatzgruppen den Standpunkt, die deutschen Gemeinden sollten vorerst in einer Zone religiösen und weltanschaulichen Niemandslands belassen werden, bevor man sie in das nationalsozialistische Gedankengut einweihen könnte. Nach Kenntnis der Lage wandten sie sich gegen eine verfrühte antireligiöse Propagandakampagne unter diesen Deutschen: „Da ihr Seelenzustand nach mehr als zwanzigjährigem Terror weitere Erschütterungen zweifellos nicht zuläßt, ist es unbedingt zu vermeiden, sie mit der religiösen Auseinandersetzung im Reich in Berührung zu bringen. Es wäre nicht wünschenswert, diese Menschen, die zum ersten Mal Lebenshoffnung geschöpft haben, mit dem (sic) üblichen noch nicht ausgereiften Streitfragen des Reichsdeutschtums bekanntzumachen "62. Demgegenüber galt die Anordnung, ,jetzt schon deutliche Hinweise (zu geben), daß in Deutschland der Führer, die Bewegung, der Staat die Menschen führen, und daß konfessionelle Zwietracht der Vergangenheit angehöre"63.

\footnotetext{
${ }^{58}$ Ereignismeldung UdSSR Nr. 86, 17.9. 1941, S.29-35.

59 Ereignismeldung UdSSR Nr.75, 6.9. 1941.

60 Ereignismeldung UdSSR Nr.103, 4.10. 1941.

${ }^{61}$ Nbg. Dok. L-221, S.6.

${ }^{62}$ Ereignismeldung UdSSR Nr. 86, 17.9. 1941, S. 35.

${ }^{63}$ Ereignismeldung UdSSR Nr.108, 9.10, 1941, S. 23.
} 
Mit der Frage des religiösen und konfessionellen Traditionalismus dieser Menschen war auch die ihrer weltanschaulichen Formbarkeit verbunden. Die Prognosen der Einsatzgruppen waren keinesfalls optimistisch. Die Vorstellungen von Deutschland waren - ihren Berichten zufolge - in dieser Bevölkerung noch allzu unklar ${ }^{64}$; der „Führer" sei der Mehrheit von ihnen nicht einmal dem Namen nach bekannt ${ }^{65}$; das „politische Bewußtsein und Beurteilungsvermögen“ seien in der Mehrheit der deutschen Bevölkerung gering. Von einer dünnen Funktionärsschicht deutscher Herkunft abgesehen, habe die „Restgruppe der politisch ungefährlichen Volksdeutschen ... zumindest ein vollkommen verzerrtes Bild von den Verhältnissen im Reich und von der Nationalsozialistischen Führung "66. Selbst wenn sie den Extremen der sowjetischen Berichterstattung über Deutschland keinen Glauben geschenkt habe, habe sie in vielen Fällen zumindest den Berichten über die Lage der Arbeiterschaft in Deutschland geglaubt. Im Bewußtsein weiter Kreise der Bevölkerung sei ferner die Gestapo dem NKWD gleichgesetz $t^{67}$, eine Vorstellung, gegen die schärfstens vorgegangen werden müsse. Im Frühjahr 1942 äußerte die Einsatzgruppe C Mißbehagen darüber, „wie wenig deutschbewußt und wie gemeinschaftsfremd diese Personen sind. Nur wenige von ihnen werden nach gründlicher Auslese und Schulung geeignet und würdig sein, als Reichsbürger anerkannt zu werden" ${ }^{\text {"68 }}$. Als erste wichtige Maßnahmen empfahlen die Einsatzgruppen daher die Stärkung des Gemeinschafts- und Volkstumsbewußtseins dieser Deutschen.

Als ein Beispiel für das ungenügend entwickelte Volkstumsbewußtsein der Deutschen in den besetzten Teilen der UdSSR wurde wiederholt ihre „vorwiegend indifferente Haltung " gegenüber den Juden erwähnt ${ }^{69}$; bezeichnend sei in dieser Hinsicht die Tatsache, „daß die Volksdeutschen nach dem Einmarsch der deutschen Truppen gegen die verbliebenen Juden keinerlei Maßnahmen ergriffen und sie als harmlose und ungefährliche Menschen bezeichneten“. Zwar sei - laut Einsatzgruppenbericht - im Altreich die Situation im Jahre der Machtergreifung nicht wesentlich besser gewesen. Doch hätten dort nach 1933 sprunghafte Verbesserungen stattgefunden. Demgegenüber habe man es nach den bisherigen Beobachtungen im Falle der südrussischen Deutschen mit einer Volksgruppe zu tun, deren „Durchdringung ... mit den einfachsten politischen Gedankengängen einen langen Zeitraum in Anspruch nehmen“ wer$\mathrm{de}^{70}$.

Allerdings waren die weiteren Maßnahmen der Einsatzgruppen geeignet, das traditionell gute Verhältnis der Deutschen zu ihrer andersethnischen Umgebung nachhaltig, wenn nicht unwiederbringlich zu verschlechtern.

Die ersten Schritte der Betreuung der Volksdeutschen zielten auf ihre „wirtschaftli-

\footnotetext{
${ }^{64}$ Ereignismeldung UdSSR Nr. 85, 16.9.1941, S. $16 \mathrm{ff}$.

${ }^{65}$ Ereignismeldung UdSSR Nr. 104, 5.10. 1941.

${ }^{66}$ Ereignismeldung UdSSR Nr. 103, 4.10. 1941.

${ }^{67}$ Ereignismeldung UdSSR Nr. 81, 12.5. 1941, S. 20.

${ }^{68}$ Ereignismeldung UdSSR Nr. 187, 30.3.1942.

${ }^{69}$ Ereignismeldung UdSSR Nr.104, 5.10. 1941.

70 Ebenda.
} 
che Sicherstellung" $a b^{71}$. Deutsche Familien wurden mit „reichlichen Beständen an Lebensmitteln“ versorgt. Der deutschen Bevölkerung wurden „Beutevieh, Beutepferde und Beuteerntemaschinen" überlassen, die vor allem aus den von den deutschen Truppen eingeholten und vom SD beschlagnahmten Abtransporten der Kolchosgüter bestanden. Weinberge, Gärten und „geräumte" Häuser wurden den Deutschen überlassen. Deutscher Immobilienbesitz, der beschlagnahmt oder unter Zwang veräußert worden war, wurde zurückgegeben, die derzeitigen Bewohner oder Besitzer vertrieb man entschädigungslos. Deutsche Familien erhielten Baumaterial und Holz zur Ausbesserung und Instandsetzung ihrer Häuser. Gleichzeitig wurde die Not in den deutschen Siedlungen durch „Verteilung des Judenguts und Judeninventars" behoben"2. Wohnungen und Wohnungseinrichtungen, Kinderbekleidung, Kinderbetten und andere notwendige Gebrauchsartikel aus dem Besitz „abgeschobener“ Juden sollten die Lebenslage der Deutschen verbessern. Zu bedenken ist, daß die Liquidation der Juden in den ersten Monaten der Besatzung unter dem Vorwand ihrer „Umsiedlung“ vollzogen wurde; nach den Aussagen von H.H.Schubert, des Adjutanten Ohlendorfs, im Nürnberger Prozeß war „Umsiedlung“ in den ersten Monaten des Rußlandfeldzugs der Deckname für die Exekution der Juden. Die Deutschen, die jüdische Wohnungen bezogen, wußten zunächst nicht in jedem Falle, worin das Schicksal der „umgesiedelten“ Vorbesitzer bestand. Je länger die Einsatzkommandos in den entsprechenden Gebieten operierten, desto geringer war allerdings die Möglichkeit, von diesem Wissen verschont zu bleiben. Die moralische Rückwirkung auf die deutsche Bevölkerung blieb nicht aus.

Die weiteren Maßnahmen der Einsatzgruppen gehörten zum Bereich der sog. sicherheitspolizeilichen Betreuung der Volksdeutschen. Als erste "Schutzmaßnahme" für die deutschen Ortschaften wurde der volksdeutsche Selbstschutz aufgestellt. Der Selbstschutz sollte aus „verläßlichen“ deutschen Männern bestehen. Diese wurden in der Regel mit „Beutewaffen“ - ausgerüstet und erhielten eine erste militärische Unterweisung. Die Instruktionen erteilten in den ersten Wochen und Monaten Angehörige der Einsatzkommandos oder ein von ihnen eingesetztes Mitglied der Wehrmacht. In den späteren Monaten wurden spezielle Ausbildungslager eingerichtet, in denen die Selbstschutzleute über mehrere Wochen hin eine militärische Grundausbildung erhielten. Zur Ausbildung gehörte auch die politische Schulung. Nach offiziellen Berichten der Einsatzgruppen stellte der Selbstschutz die Wachen, Wegekontrollen und Patrouillen. Er leistete Hilfsdienst bei Registrierungen Volksdeutscher und Fremdvölkischer, bei Haussuchungen und Befragungen. In der Praxis reichte die Tätigkeit der Selbstschutzleute in vielen Fällen weiter.

Nach Eintreffen in einer deutschen Ortschaft bestimmten die im Durchschnitt 5 bis 8 Mann eines Einsatzkommandos ein Haus zur „Kommandantur“ und zum Quartier. Der Leiter des Einsatzkommandos nannte sich „Kommandant“. Er bestätigte dann entweder den bereits von der Wehrmacht eingesetzten Bürgermeister oder ernannte

\footnotetext{
${ }^{11}$ Ereignismeldung UdSSR Nr. 108, 9.10. 1941, S. 21-24.

72 Ereignismeldung UdSSR Nr.103, 4. 10. 1941, Nr. 108.
} 
einen neuen. Der Bürgermeister gab dem Kommandanten die für den Selbstschutz geeigneten Männer und Burschen an (in den ersten Monaten wurden sie aus der Altersgruppe von 15 bis 45 Jahre ausgewählt, später wurden systematisch die Jahrgänge 1914-18 einberufen) und beorderte sie zur Kommandantur. Hier versah man sie mit Armbinden und - wenn vorhanden - mit Gewehren und informierte sie über ihre Aufgaben. In Dörfern von 1000 bis 5000 Einwohnern wurden in der ersten Zeit meist bis zehn, später, nach der Ankunft der SS-Sonderkommandos der Vomi, alle Männer der betreffenden Jahrgänge eingezogen. Während der Anwesenheit der Einsatzkommandos am Ort wurden sie als orts- und sprachkundige Hilfen und ausführende Organe der Einsatzgruppen verwendet. Nach vollzogener Aktion und Abzug der Einsatzkommandos blieb meist ein Mann des Kommandos am Ort zurück, „um der Inschutznahme aller deutschen Dörfer sichtbaren Ausdruck zu verleihen "73. Die Tätigkeit des Selbstschutzes beschränkte sich dann in der Hauptsache auf Aufrechterhaltung von "Sicherheit und Ordnung“, was sehr oft die Abwehr von Partisanenangriffen bedeutete. Neue Aufgaben fielen dem Selbstschutz mit Eintreffen der SS-Sonderkommandos der Vomi zu.

Während der Anwesenheit der Einsatzkommandos am Orte mußten die Selbstschutzleute (nach Berichten der Einsatzgruppen) „zur Stärkung der Autorität des Bürgermeisters“ beitragen; sie mußten „unzuverlässige Elemente“ anzeigen, „örtliche Mißstände“ abstellen helfen und die „Durchkämmung in politischer Hinsicht" durchführen. Mit ihrer Hilfe wurden mehrsprachige Schilder gefertigt, welche die entsprechenden Dörfer als volksdeutsche Orte kennzeichneten und folgende Aufschrift trugen: „Die Volksdeutschen stehen unter dem Schutz der deutschen Wehrmacht. Wer sich an ihnen und ihrem Besitz vergreift, wird erschossen."74 Die Selbstschutzleute überwachten ferner die Fertigung von Hakenkreuzfahnen für jedes Haus, den Kirchturm und die öffentlichen Gebäude. Sie leisteten den Einsatzkommandos vor allem wichtige Dienste bei der „Freimachung des Gebietes von Juden und kommunistischen Elementen"75.

Um zu verdeutlichen, wie dies in der Praxis vor sich ging, sei das Beispiel des Einsatzkommandos 12 der Einsatzgruppe $D$ herausgegriffen ${ }^{76}$. Nachdem bereits mehrere EKs der Einsatzgruppen $\mathrm{D}$ die deutschen Siedlungsgebiete auf dem Boden des früheren Gouvernements Cherson durchkämmt hatten, traf Ende August als letztes das EK 12 im Schwarzmeerraum ein. Von seinem Standort Nikolajew aus setzte es die von den vorangegangenen EKs begonnene „Betreuung“ der dichten deutschen Sied-

${ }^{73}$ Ereignismeldung UdSSR Nr. 108, 9.10. 1941, S. 21.

${ }^{4}$ Vgl. Werner, P., Ein Schweizer Journalist sieht Rußland; auf den Spuren der deutschen Armee zwischen San und Dnjepr, Olten 1942, S. 167.

${ }^{75}$ Ereignismeldung UdSSR Nr. 108, 9.10. 1941, S. 22.

${ }^{76}$ Nach den Vernehmungsprotokollen im Ermittlungsverfahren gegen D. und A.K. wegen Mordes (NSG), Staatsanwaltschaft München 1, Az.: 119 Ks 6/70 und 119 c Js 1/69. Die in Anführungszeichen wiedergegebenen Ausdrücke wurden von den Zeugen, Volksdeutschen der entsprechenden Ortschaften, gebraucht. Zu Weg und Aktionen der Einsatzgruppe D vgl. Krausnick, Einsatzgruppen, S. 195-205. 
lungsgebiete westlich des Bug fort. Besonders aktiv waren dabei zwei im deutschen Ort Speyer stationierte und das Berezaner Gebiet kontrollierende sog. Restkommandos. Die Ablösung des EKs durch die SS-Kommandos der Vomi erfolgte spätestens Anfang Oktober 1941. Der verhältnismäßig kurze Einsatz des EK 12 in diesem Gebiet bot dennoch Zeit genug für Ungeheuerliches, die massenweise Liquidation der hier lebenden oder auf der Flucht durchziehenden Juden ${ }^{77}$ (wobei auch andere EKs beteiligt waren). In den volksdeutschen Gebieten hatten die EKs um diese Zeit bereits den Namen „Aufräumkommandos“ erhalten. Insbesondere war es nach Augenzeugenberichten überall bekannt, daß „die Juden von den Deutschen erschossen werden“.

Die Aktionen verliefen nach einem bestimmten Schema. Nachdem sich das EK meist in privaten Häusern - einquartiert hatte, verlangte der Kommandant vom Bürgermeister eine Liste der ortsansässigen Juden; in größeren Orten wurden die jüdischen Familien durch Maueranschlag aufgefordert, sich zur „Umsiedlung“ zu melden. Auf Anweisung des Kommandanten schickte der Bürgermeister dann Selbstschutzleute in die jüdischen Häuser, um die Familien „zum Bürgermeisteramt zu holen“. Der mit Armbinden versehene, bewaffnete Selbstschutz eskortierte die Juden zu Fuß (und in einigen Fällen in russischer Kommissarpose beritten) zum Amt. Auf dem Weg durch die Ortschaft, den viele der jüdischen Familien weinend und wehklagend zurücklegten, begegneten dem Elendszug zahlreiche deutsche Mitbewohner, andere Deutsche kamen aus ihren Häusern auf die Straße. Die Szene wurde teils mit Neugier - so von den Dorfkindern -, teils mit Scham und Bedauern verfolgt. In vielen Fällen flehten die jüdischen Familien ihre deutschen Nachbarn um Hilfe und Fürsprache an. (Es kam vor, daß durch die Intervention der deutschen Bevölkerung ein Aufschub erreicht wurde; so konnte im Ort Waterloo mit ca. 2000 deutschen Einwohnern und drei jüdischen Familien, von denen zwei geflohen waren, durch mehrmaliges Eintreten der Volksdeutschen für die verbliebene jüdische Familie Moische die Aussetzung der Exekution bis zum Abzug des Kommandos durchgesetzt werden.) Vom Bürgermeisteramt oder einem anderen Sammelplatz aus wurden die jüdischen Einwohner ihrer sozialen Herkunft nach Handwerker, Lehrer, meist aber wie die Deutschen Kolchosbauern - zur Exekutionsstelle geführt, wo sie bereits die Männer des motorisierten Einsatzkommandos erwarteten. Am Rand einer Vertiefung (Viehfriedhof, Silo, Mulde, Torfplatz usw.) mußten die Juden ihre Oberbekleidung ablegen. Dann wurden sie mit dem Gesicht zur Grube erschossen. Die Selbstschutzleute schütteten die Gruben zu.

In anderen Fällen wurden die jüdischen Familien über Nacht in größeren Räumen (Stallungen, Kirchen, Kellern des Bürgermeisteramts oder „Verschlägen“) festgehalten; die Bewachung übernahm auch hier der Selbstschutz. Gelang es Eingesperrten zu entkommen, so nahm der Selbstschutz die Verfolgung auf. In einigen Fällen wur-

\footnotetext{
7 Vgl. dazu die Urteile gegen vier Angehörige des EK 10 a der Einsatzgruppe D, das ebenfalls im volksdeutschen Gebiet vor Speyer operierte: Landgericht München 1. Urteile in der Strafsache gegen E.B., H.D., O.-E.P., W.S. vom 23.3. 1973, IfZ, Gm 07.77.
} 
den die Gejagten „auf der Flucht erschossen“, in anderen unter Schlägen und Mißhandlungen zu ihren Verwahrungsplätzen zurückgeführt.

Des öfteren beschränkte man sich nicht auf die Liquidierung der ortsansässigen Juden. Unter der Leitung der EK-Männer wurden vom Selbstschutz die umliegenden Gebiete „durchkämmt" und die auf der Flucht befindlichen Juden „gefangen“. Man hielt sie ebenfalls die Nacht über fest. Oft hatten dann am nächsten Morgen deutsche Bauern mit Fuhrwerken zu erscheinen, um die Juden zur Exekutionsstelle zu transportieren, nicht selten mehrere „Fuhren Juden“" an einem Tag. Waren die am Rand des Orts gelegenen Vertiefungen nicht ausreichend breit und tief, fiel es dem Selbstschutz zu, die Gruben „mit Spaten und Schaufeln“ auszuweiten. Die Selbstschutzleute waren auch anwesend beim Eintreffen der "Judenfuhren" - oft genug erkannten sie darauf Nachbarn, Bekannte oder Schulfreunde. Aufgabe des Selbstschutzes war es schließlich noch, bei den Erschießungsvorbereitungen die Anweisungen der EKMänner zu übersetzen. Drohten Selbstschutzleute „weich“ zu werden, so schickten die SD-Leute sie für die Zeit der Erschießung „zur Seite“. Danach mußten sie an den vielfach blutbespritzten Orte zurückkehren, um die Masse der sich noch bewegenden und oft röchelnden Körper mit Erde zu bedecken („Der Anblick war für mich furchtbar. Ich konnte zwei Tage nicht essen." A.F. aus Hoffnungsthal). In wenigen Fällen meldeten sich einzelne Selbstschutzleute "immer freiwillig zur Judenerschießung“ (so ein gebürtiger Landauer in Speyer). In anderen Fällen forderten die EK-Männer Selbstschutzangehörige zur Durchführung der Erschießungen auf. Diese kamen den Anordnungen in der Regel nach, wenn auch unwillig und mit anhaltender Verstimmung. In Landau, einem Ort mit etwa 4-5000 Deutschen und einigen jüdischen $\mathrm{Fa}$ milien, wurde Mathias Hegel, der Leiter der Selbstschutzabteilung des Ortes, aufgefordert, die Erschießungen durchzuführen. Er erklärte, daß er „dies nicht könne“. Dem Drängen des EK-Kommandanten setzte er entgegen, daß man ihn dann besser zuerst „ins Loch schießen solle“. Daraufhin nahm der Leiter des Kommandos selbst die Erschießungen vor.

In dieser Zeit fand (nach Berichten der ortsansässigen Deutschen) in den Siedlungen "das große Judensterben“ (Speyer) statt. „Es knallte immer" (Waterloo). Auch jüdische Altersheime wurden liquidiert (Speyer, Landau), wobei ein deutscher Pförtner sich zunächst weigerte, die Gruben auszuheben. In Freiberg, einem Ort in der Nähe von Hoffnungsthal, waren 1928350 Juden angesiedelt worden. Nach ihrer Liquidierung wurden hier nun deutsche Familien „aufgesiedelt“.

Die zahlenmäßig größten der „Judenaktionen “ waren die „Flüchtlingsaktionen“, wobei „endlose Wagenkolonnen“ jüdischer Flüchtlinge, zumeist aus Rumänien und Bessarabien kommend, von den Kommandos „eingefangen“ wurden. Die deutschen Selbstschutzleute waren am „Judentreiben" beteiligt und „lieferten die Juden beim Bürgermeisteramt ab“. Zu Hunderten wurden die Flüchtlinge in Scheunen, Ställen, Kirchen und Gemeindehäusern gefangengehalten und von den Selbstschutzleuten bewacht. Bei Ausbrüchen oder Flucht wurde die Kirchturmglocke dreimal geschlagen, und der zusammenströmende Selbstschutz ging auf „Judenjagd“. Am nächsten Morgen oder nach Verlauf mehrerer Tage wurden die jüdischen Flüchtlinge wie üb- 
lich auf Fuhrwerke der Ortsansässigen verladen und unter Bewachung des Selbstschutzes zu den „Mulden“, „Panzergräben“, vom Regen ausgewaschenen Rinnen oder zu den zuvor ausgehobenen Gruben gefahren. So wurden in der Rinne des Nikolajew-Damms bei Speyer 800 bei Katharinenthal aufgegriffene Juden erschossen. Bei größeren Aktionen dieser Art sammelte der Selbstschutz Kleider und Schuhe der Erschossenen ein und verteilte sie an bedürftige und notleidende Deutsche. Die zum Teil mit Blut beschmierten Kleider wurden „Juddekleider“ genannt („sie waren oft besser als unsere"). - Nach dem Massaker im Babij Jar in Kiew (29.-30. September 1941), wo 33771 Juden erschossen wurden, kamen 139 Wagenladungen von „Judenkleidern" unter den Deutschen der Ukraine zur Verteilung ${ }^{78}$.

Überstieg die Zahl der „aufgefangenen Judentrecks“ das Fassungsvermögen der Bodensenken, wurden die Juden in „Judenzügen“ in die nächstgrößeren Vernichtungszentren getrieben, so z. B. mehrere tausend Juden von Okna nach Dubossary. Die "Treibwege" betrugen oft 60-70 km. Auch hier war der Selbstschutz aktiv. Unter anderem trieb er mehrere hundert Juden von Sophienthal nach Dubossary. Andere „Judenzüge" wurden "nach Speyer abgeliefert". Aus Kassel, einem Ort mit ca. 10000 Deutschen, wurden die gefangenen Juden "auf volksdeutschen Fuhrwerken“ nach Speyer gebracht. In Zentren wie Speyer und Dubossary wiederholten sich solche Vorgänge „alltäglich“. Einige Kolonnen jüdischer Flüchtlinge versuchten, über den Bug zu gelangen. Ihre „dort herumstehenden Fahrzeuge und Wagen" haben die Volksdeutschen und unter ihnen die Selbstschutzleute „an sich genommen“. Auch „Ausplünderungen“ von flüchtenden Juden wurden berichtet.

Wirksamer Widerstand gegen solche Aktionen wurde von seiten der örtlichen deutschen Bevölkerung nicht geleistet. Milde Formen von Widerstand hingegen kamen nach den Berichten hin und wieder vor. So verwehrte eine Frau dem bei ihr einquartierten EK-Mann den Eintritt in das Haus ,in diesen blutbeschmierten Stiefeln“. In Katharinenthal hielt Barbara Haaf (verschollen) einem EK-Mann vor: „Was ihr dort macht, ist nicht schön, worauf der Deutsche erwiderte, daß dies sein müsse".

Nach Aussage von E.E. Bieberstein (eigentlicher Name Schymanovsky, evangelischer Theologe und Leiter des EK 6 der Einsatzgruppe $C$ mit Standort Kiew) zeichneten sich die Deutschen aus der Sowjetunion bei den Erschießungen durch eine „Blutgier“ aus, die selbst die SS-Leute erschreckte ${ }^{79}$. Die Vernehmungs- und Untersuchungsprotokolle der Einsatzgruppen- und Selbstschutzprozesse zeigen jedoch, daß sich die Zahl der freiwillig und aktiv mit den EKs kollaborierenden Deutschen in einem sehr begrenzten Rahmen hielt. Ihre Namen wurden von ihren Mitbürgern mit deutlicher

${ }^{78}$ IMT, Bd.XXI, Fall XII, Nbg. Dok. NOKW-3147; vgl. dazu Reitlinger, G., The Final Solution. The Attempt to Exterminate the Jews of Europe, 1939-1945, London 1953, S. 234, 564.

79 Auf die Frage, wer die Erschießungen durchgeführt habe, antwortete Bieberstein: „Vor allem die SS-Männer ..., auch eine ganze Reihe von Volksdeutschen ... Es war so, daß die Volksdeutschen in Rußland auf die Bolschewisten eine Wut hatten. Es waren Studenten dabei, die durchgemacht hatten, wie ihre Eltern erschossen wurden. Das hat uns direkt erschreckt, was die für eine Blutgier hatten." E. E. H. Bieberstein, Interrogation Nr. 1499-A, 29.6. 1947, Nbg. Dok. NO-4997; vgl. auch Hilberg, R., The Destruction of European Jews, Chicago 1961, S. 206. 
Distanz und gelegentlich mit Abscheu genannt. Solche Männer schlossen sich den EKs in einigen Fällen als Hilfspolizisten an und verließen ihre Heimatorte. $\mathrm{Zu}$ ihnen gehörten die 1964 in der UdSSR zum Tode verurteilten Deutschen Alfons J. Gezfrid (Götzfried oder Hetzfried), geboren 1919 in Rastatt, Gebiet Odessa (Hilfspolizist des Sonderkommandos 11 b der Einsatzgruppe D), und Ivan (Johann) D. Girt (Hirt), geboren 1924 im Kirchdorf Mamals, Kreis Simferopol, Krim (ebenfalls Sonderkommando 11 b). Beide dienten in der von W. Kehrer auf der Krim zusammengestellten „Tatarischen Division“, die auch der Einsatzgruppe D unterstand. Außer bei Massenerschießungen waren sie später beim Einsatz der zur Massentötung verwendeten Gaswagen (russisch: dušegubcy) beteiligt ${ }^{80}$.

Die breite Bevölkerung in den deutschen Siedlungsgebieten reagierte auf die Massenvernichtungen (bis Ende September 1941 waren im Operationsbereich der Einsatzgruppe D 35782 Juden liquidiert worden ${ }^{81}$ ) mit Passivität, wenngleich sie die Aktionen mißbilligte und oft Mitleid empfand. Der bei einer Erschießung anwesende Selbstschutzmann G.H. aus Bergdorf: „Es war schrecklich für uns, das alles mitanhören zu müssen, zumal wir diese Leute gut kannten.“ A. B., Mitglied des Selbstschutzes in Waterloo: „Es tat uns allen furchtbar leid, denn wir kannten die Juden ja sehr gut. Es war mir unverständlich, warum sie damals erschossen wurden. Wir sahen es alle als eine Schweinerei an, aber was sollten wir machen." Der in Waterloo eingesetzte Bürgermeister K.: „Ich konnte keinerlei Einflüsse auf die Geschehnisse, die sehr schrecklich waren, ausüben." Eine gewisse Erklärung für diese passive Haltung der deutschen Bevölkerung lag in der durch die vorausgegangenen Terrorjahre bedingten Furcht, dem Wunsche, unterzutauchen, nicht aufzufallen, sowie in einer gewissen moralischen Verwirrung der Werthaltung und Einstellung gegenüber dem Mitmenschen. H.B. aus Waterloo führte das Schweigen der ortsansässigen Deutschen darauf zurück, „daß man auch unter der deutschen Zeit möglichst verborgen bleiben wollte, denn wir alle hatten ja das Empfinden, daß das Unrecht war, was da geschah". P.S. aus Waterloo: „Für meine Begriffe war das, was die SD-Leute in Waterloo gemacht haben, Unrecht, daran gibt es keinen Zweifel. Wir konnten es aber nicht verhindern, weil wir selbst Angst vor diesen Leuten hatten und auch gar nicht den Einfluß besaßen, der nötig gewesen wäre, um gegen dieses Vorgehen mit Aussicht auf Erfolg einschreiten zu können." Der in Waterloo während der ganzen deutschen Besatzungszeit als Bürgermeister fungierende K. befand später: „... die Kriegsereignisse hatten uns nicht besonders berührt. Aber die Erschießungen von Menschen, die niemandem von uns etwas zu Leide getan hatten, riefen doch einen tiefen Eindruck hervor. Wir alle begrüßten die deutschen Soldaten als unsere Befreier. Wir alle waren froh, daß sie nun da waren, und glaubten uns von allem Bösen befreit. In der deutschen Nation sahen wir ... immer das Beste, nur das Gute und das Vorbild. Nun aber waren sie da, unsere Landsleute, und erschossen für uns ohne Grund unsere Nachbarn, mit denen

${ }^{80} \mathrm{Vgl}$. Vernehmungsprotokolle russischer Zeugen im Ermittlungsverfahren gegen W.K. wegen Mordes (NSG), Bayrisches Landeskriminalamt, III a/SK K 5563 - Tgb.Nr. 447/60, Staatsanwaltschaft München I, Az.: $115 \mathrm{Js}$ 42/66.

${ }^{81}$ Krausnick, Einsatzgruppen, S. 201. 
wir friedlich gelebt hatten. Das war uns allen unverständlich, und wir empfanden es wie einen Schlag ins Gesicht. Es war ein Schock für uns, dieses Volk, dem wir angehörten, ständig als leuchtendes Vorbild des Guten vor Augen gehabt zu haben."

Nicht weniger bedrückend war es für viele Deutsche der UdSSR, daß zugleich mit den "Judenaktionen“ auch zahlreiche Deutsche aus ihren eigenen Reihen liquidiert wurden. In Übereinstimmung mit den Anweisungen Himmlers und Heydrichs wurden auch Volksdeutsche „exekutiert“, wenn sie als ehemalige Funktionäre, erklärte oder verdächtige Kommunisten, „radikale Elemente“, „Heckenschützen“ (Partisanen), Hetzer u.s. w. bekannt waren oder als solche denunziert wurden. In der Praxis genügte ein Wort eines neidischen Nachbarn, ja die Denunziation eines Kindes, um einen Deutschen standrechtlich zu erschießen. Nach wiederholten Aufforderungen zur Denunziation wurden von der deutschen Bevölkerung meist jene Deutschen namhaft gemacht, von denen man annahm, daß sie in den Jahren des Stalin-Terrors mit dem NKWD zusammengearbeitet hätten und für Verschleppungen der Mitbürger verantwortlich seien. Beweise konnten und brauchten nicht erbracht zu werden. Auch anderes "unkorrektes" Verhalten war ein ausreichender Grund zum Erschießen. Selbst Denunziationen, die auf reiner Mutmaßung und blindem Verdacht beruhten, trafen ihr Ziel. Die Einsatzkommandos sahen es dabei als ihre Aufgabe an, „das Vertrauen der volksdeutschen Bevölkerung weiterhin dadurch (zu stärken), daß notfalls auch gegen Volksdeutsche mit den schärfsten Maßnahmen eingeschritten

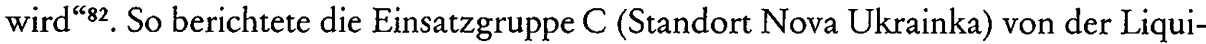
dierung zweier Deutscher, „übler bolschewistischer Hetzer“, in der deutschen Siedlung Rosa Luxemburg. In Stara Buda, Bezirk Schitomir, sei auf „Bitte“ der Bevölkerung die „Unschädlichmachung“ des Kommunisten A. Grünwald vorgenommen wor$\mathrm{den}^{83}$. Einsatzgruppe D (Standort Nikolajew) unterschied nach eigenen Aussagen in den deutschen Dörfern „zwei Kategorien“ von deutschen Funktionären ${ }^{84}$ : Die erste Kategorie habe die Anweisungen nur dem Buchstaben nach ausgeführt und nach Möglichkeit versucht, ihre „Volksgenossen“ zu schützen. „Sie wurden deswegen häufig mit Gefängnis bestraft. Derartige Volksdeutsche wurden in Landau, Worms und außerhalb des Berezaner Gebiets in Wilhelmsfeld und Marienberg angetroffen." Die zweite Kategorie der deutschen Funktionäre habe „dem ausgesprochenen Proletariertyp" angehört, die Anweisungen blind befolgt und, „um sich die Gunst der ihnen geistig überlegenen Vorgesetzten zu erwerben, die Volksdeutschen mit allen möglich(en) Mitteln bedrängt“. Sie seien dabei oft zu wahren Tyrannen in ihrem Bezirk geworden. Den letzten galten offiziell die Vergeltungsakte der EKs. In der Praxis erschossen sie ortsansässige Deutsche aus einer Vielzahl von Gründen. So wurden von den Angehörigen des EK 12 der Einsatzgruppe $D$ in Neudorf, einem Ort von etwa 2000 deutschen Einwohnern, die als Kommunisten verdächtigten Männer August Weiß, Jakob Schlichte, Friedrich Rohrbach und Jakob Bonnet im Keller der Kolcho-

\footnotetext{
${ }^{82}$ Ereignismeldung UdSSR Nr. 80, 11.9. 1941, S.12.

${ }^{83}$ Ereignismeldung UdSSR Nr. 86, 17.9. 1941, S. 33.

${ }^{84}$ Ereignismeldung UdSSR Nr. 104, 5. 10. 1941.
} 
se „festgesetzt" und dann zusammen mit den jüdischen Familien Bowik (oder Bopik) und Narianskij an der „Grube“ erschossen. Selbstschutzleute nahmen die Festnahmen vor und stellten die Bewachungskommandos. Im selben Ort wurde Fritz Müller, der frühere Vorsitzende des Dorfsowjet, selbst erst kürzlich aus sowjetischer Haft entlassen, während der deutschen Besatzungszeit gefangengehalten und vor Abzug der deutschen Bevölkerung erschossen. In Landau wurde der Direktor der Mittelschule, Bach, zusammen mit Eva Pfaff, Rosa Schäfer und der jüdischen Frau (Zimbelmann) des Deutschen Gimpel erschossen. Im Falle der deutschen Frauen lag - nach Aussagen - eine persönlich begründete Denunziation zugrunde („Racheakt“). In Bergdorf wurden der Deutsche Weiß aus Neudorf und die Ortsansässigen Friedrich Jesser und Friedrich Stroh aus unbekannten Gründen erschossen. Der deutsche Lehrer Volz war mit seiner jüdischen Frau (Lehrerin) und seinen zwei Kindern zunächst im Gemeindehaus eingesperrt worden. Nach einem Fluchtversuch wurde die ganze Familie zusammen mit den Bergdorfer Juden erschossen. In Glücksthal wurden sieben deutsche Männer, unter ihnen Johann Vögele, Jerges und Bratzel, für Verschleppungen ihrer Mitbürger verantwortlich gemacht und zusammen mit der Familie des jüdischen Glasers erschossen. In Worms, einem Ort von ca. 3500 Deutschen, wurden der frühere Bürgermeister Georg Walter sowie Emmanuel Knodel und Frau verhaftet und zum Friedhof geführt. Hier mußten sie zusammen mit den fünf jüdischen Familien des Ortes selbst eine Grube schaufeln, in die sie dann „hineingeschossen“ wurden. In Friedensthal wurden der frühere Kommunist Albert Schmidt sowie, aus unbekannten Gründen, die Männer Meier, Alexander und Engel liquidiert. Der frühere Gemeindeschreiber Engel wurde dabei nach gewaltsamen Verhören an einem Baum vor der Kommandantur stranguliert. Aus Waterloo verschwand Frau Ziegler zur „Umsiedlung“. In Hörnersfeld wurde Linus Wetzstein, ein Trinker und Pelzhändler, der „russische Bekannte" hatte, erschossen. In Rohrbach wurden vier Deutsche, Bolländer ${ }^{85}$, Hust, Herzel und Czisgenow, erschossen.

Maßnahmen, wie die hier vom EK 12 der Einsatzgruppe D durchgeführten, waren für alle Einsatzgruppen in den besetzten Gebieten der UdSSR charakteristisch. Die Liste der Fälle ist noch offen, denn die Ermittlungen in den Einsatzgruppen- und Selbstschutzprozessen halten an. Die Aktivität der Einsatzgruppen Heydrichs versetzte die deutsche Bevölkerung, die eben glaubte, nach dem Terror der Stalin-Zeit aufatmen zu können, in neue Furcht. Doch mit dem Abrücken der Einsatzkommandos waren die Schrecken in vielen Fällen noch nicht beendet: die nachrückenden SSSonderkommandos der Volksdeutschen Mittelstelle setzten das Werk auf wohlvorbereitetem Boden fort.

${ }^{85}$ Nach Ereignismeldung UdSSR Nr. 104, 5. 10. 1941, Bolenda. 